\title{
Status and Geographical Distribution of Indigenous and Quarantine Fruit Fly Species (Diptera: Tephritidae) in Sarawak
}

\author{
FELICIA KUEH TAI FUI*1 ${ }^{1}$, MEGIR GUMBEK ${ }^{2} \&$ SULAIMAN HANAPI ${ }^{3}$ \\ ${ }^{1,3}$ Faculty of Resource Science and Technology, Universiti Malaysia Sarawak, 94300 Kota Samarahan, Sarawak, \\ Malaysia; ${ }^{2}$ Agriculture Research Centre Semongok, Department of Agriculture Sarawak, Jln Puncak Borneo, 93720, \\ Kuching, Sarawak, Malaysia
}

\begin{abstract}
The provision of reliable pest records, one of which is fruit fly record, is one of the vital components under the International Plant Protection Convention. A fifteen-month survey to detect fruit flies using male pheromone lure trapping method was carried out from April 2010 to July 2011. The standard Steiner traps and three types of lures (ME-lure, CUE-lure and Trimed-lure) were used. The traps were placed at every five kilometre interval along the east-west trunk road of Sarawak. The geographical distribution of the fruit fly species was established through Global Positioning System mapping. Twenty species of Dacinae fruit flies which includes nine unknown Bactrocera species were detected along the east-west trunk road of Sarawak. The main species of fruit fly caught in the ME-lure trap were Bactrocera papayae, B. carambolae and $B$. umbrosa, whereas $B$. cucurbitae, B. papayae, B. caudata, B. tau, B. apicalis, B. nigrotibialis and B. albistrigata were caught in the CUE-lure trap. The quarantine species, which is species of potential economically importance in Sarawak, $B$. correcta, B. dorsalis, B. occipitalis and B. zonata were absent in both ME-lure and CUE-lure traps. The Mediterranean fruit fly (Ceratitis capitata) was not detected in the Trimed-lure trap. Out of the twelve identified species, eleven of them were detected along the main road of Sarawak. Bactrocera papayae and B. caudata were abundant, whereas the other species were more prevalent at some locations.
\end{abstract}

Keywords: survey, fruit fly, species, traps, lure, detection

\section{INTRODUCTION}

Fruit flies, belonging to the family Tephritidae, are economically important as they cause significant damage to fruits and vegetables, resulting in significant losses and high control costs (White \& Elson-Harris 1992). The family Tephritidae is further divided into subfamily Dacinae, Ceratitidinae, Trypetinae, Tephritinae and Schistopterinae (Hardy 1997). Under this classification, the Dacinae fruit flies are considered major pests of agriculture (Waterhouse 1997). The climatic condition in Malaysia enables the whole year round cultivation of the fruit fly host plants and therefore encourages the fruit fly to multiply in a rapid manner since the food source is always available. Thus, they can cause serious threat to the Malaysian fruit industry (Vijaysegaran \& Shamsudin 1991).

The importance of fruit flies in Malaysia has been highlighted since the establishment of National Agricultural Policy in 1984 (Tan 2004). Their occurrence has considerable

*Corresponding author: felicktf@sarawak.gov.my quarantine implications and poses serious problems in the export of fruit and vegetables commodities, particularly to countries where certain fruit fly species are absent (McMaugh 2005).

The provision of reliable pest records is one of the vital components under the International Plant Protection Convention. Pest record, which indicates the presence or absence of a specific pest, is essential for the importing and exporting countries to establish, maintain or verify their pest free area and produce their pest risk analysis. Therefore, a fruit fly surveillance report, reporting the status of fruit fly species, is vital for pest free assurance or low pest prevalence to the importing and exporting countries based on their quarantine regulations and requirements.

This is the first survey of Dacinae fruit fly in Sarawak. This paper reports on the types and numbers of species caught in different types of lures and their geographical distribution. 


\section{MATERIALS \& METHODS}

The surveillance procedures, following the International Standards for Phytosanitary Measure No. 6 (International Plant Protection Convention 1997) are adopted in this project. The survey was carried out in seven phases, from April 2010 to July 2011.

\section{Trap and Lure Selection}

The standard Steiner trap, which is a horizontal and clear cylinder with an opening at both ends (John 2008), was used. Three types of lures were used. They were Methyl-Eugenol lure (ME), CUE lure and Trimed lure. Each lure was targeting at certain fruit fly species. The use of Trimed-lure was meant to attract the Mediterranean fruit flies (Ceratitis capitata). Eighty four percent Malathion was mixed with the lure at a ratio of 1: 3 , to avoid the fruit flies from escaping and predation of the trapped flies. The lure was impregnated into a cotton wick hung in the center of the trap.

\section{Trapping Procedure}

The survey team comprised three persons. Each personnel handled each type of lure because it is essential that the lures did not become cross contaminated with each other and attracts the wrong targeted species of fruit fly. Suitable and proper sites at every 5 kilometre interval along the main road were selected. The traps were placed at, at least 5 metres from the east-west trunk road of Sarawak. The trapping height was about one and a half metres and distance of each trap at each site was one metre apart. The trap entrance was also cleared from twigs and leaves to allow proper air flow and easy access of the fruit fly. Global Positioning System (GPS) was used for the trap mapping. The samples at each site were collected at weekly interval while the lures were refilled fortnightly. The trappings were conducted at seven phases (Table 1) along the east west trunk road of Sarawak, from Kuching Division to Lawas Division. Each phase consists of several areas. The trappings were done one or two phases at a time. The traps were relocated every three months.

A total of 678 traps were placed at 226 locations along the east-west trunk road of Sarawak (Table 1). The traps were also fixed along the access road to Biawak, Serikin, Tebedu and Lubok Antu, which are near to the Indonesian border. These could be the points of entry of the quarantine species.

\section{Identification}

Every single sample was identified morphologically in the laboratory. The wings of fruit fly bear significant taxonomic characteristic and were extensively used for identification. Other important characters occur on the thorax, abdomen, head and legs. The main references used for the specimen identification were Handbook of identification of fruit flies in the tropics (UPM Serdang) (Rohani \& Abdul Ghani 1990) and Fruit fly of economic significance: their identification and bionomics (ACIAR and CABI) (Ian \& ElsonHarris 1992). The identified and unidentified samples were also cross checked with Department of Agriculture Semenanjung Malaysia in Kuala Lumpur.

\section{Mapping}

The GPS data was converted to Borneo Rectified Skew Orthomorphic (BRSO) data using State Geomatic Data Clearinghouse 2.0 online toolbox. The BSRO data was then sent to Soil Management Branch for mapping. The geographical distribution of the traps through GPS mapping is shown in Figure 1.

Table 1. Number of traps and area of survey.

\begin{tabular}{clcc}
\hline Phase & Area & No. of location & No. of trap \\
\hline 1 & Bau - Lundu (including Biawak and Serikin) & 28 & 84 \\
2 & Siburan - Lachau (including Tebedu) & 30 & 90 \\
3 & Lachau - Saratok (including Lubok Antu) & 42 & 126 \\
4 & Saratok - Selangau & 35 & 105 \\
5 & Selangau - Bintulu & 40 & 120 \\
6 & Bintulu - Miri & 28 & 84 \\
7 & Miri - Lawas & 23 & 69 \\
\hline & Total & $\mathbf{2 2 6}$ & $\mathbf{6 7 8}$ \\
\hline
\end{tabular}




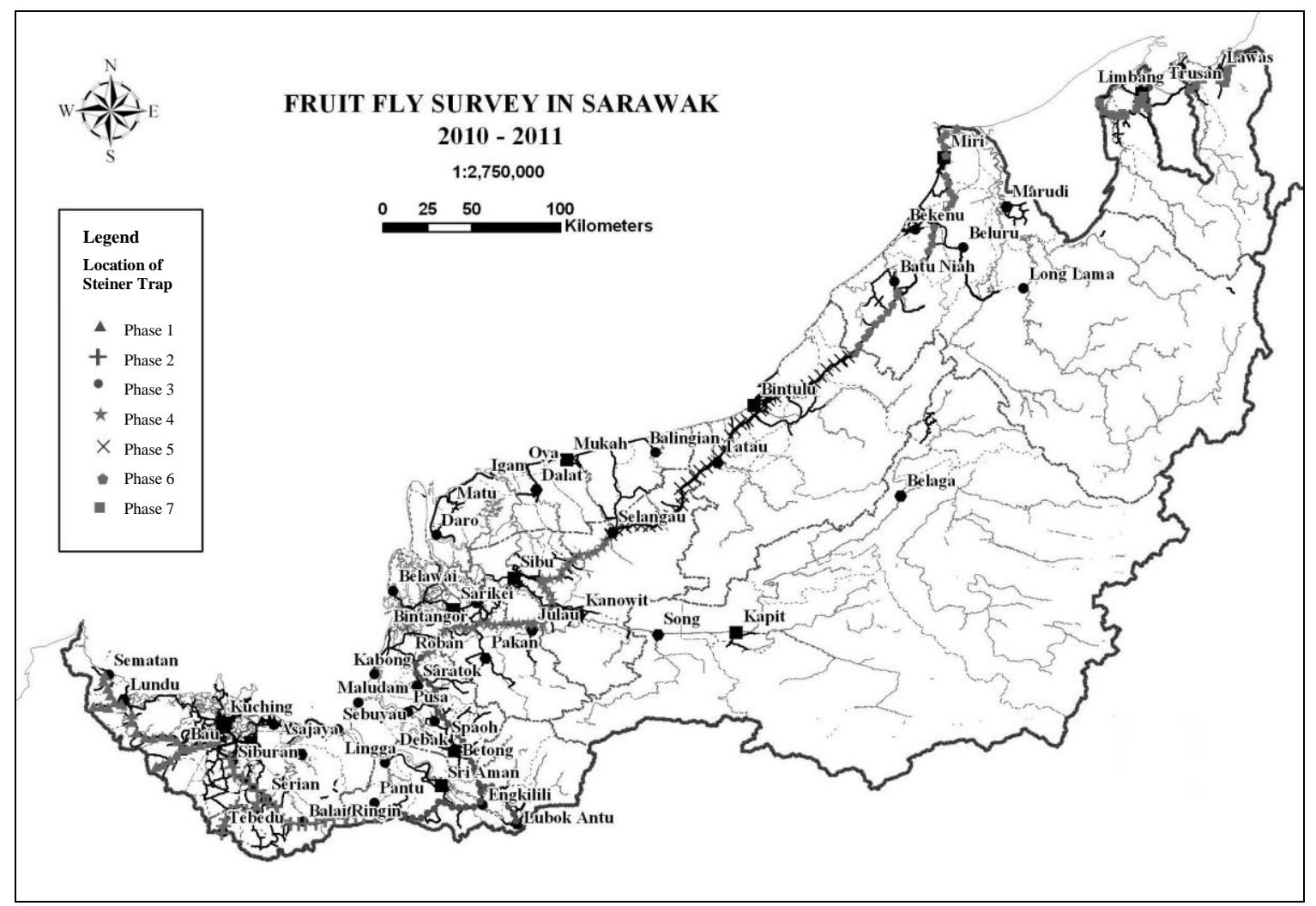

Figure 1. Location of the Steiner traps along the east-west trunk road of Sarawak.

\section{RESULTS \& DISCUSSION}

A total of 177, 505 Dacinae fruit fly samples were collected and identified (Table 2). 664 fruit flies from the other Tephritidae subfamily were also trapped but are not discussed in this paper.

Twenty species of Dacinae fruit flies which includes eight unknown Bactrocera species were detected along the east-west trunk road of Sarawak. The identified species are $B$. papayae, B. caudata, B. carambolae, $B$. cucurbitae, B. tau, B. albistragata, $B$. nigrotibialis, $B$. umbrosa, $B$. apicalis, $B$. ubiquitus, D. longicornis and D. hochii. The Bactocera species which had not been clearly identified are currently categorized under Bactrocera complex species and further identification is needed.

The highest number of fruit fly caught occurred in Phase 1 (Bau to Lundu area), followed by Phase 7 (Miri to Lawas area) and Phase 2 (Siburan to Lachau area) (Table 2). These stretches of road are along the cultivated areas with various types of fruit trees such as Psidium sp., Syzygium samarangense, Artocarpus heterophyllus, samarangense, Artocarpus heterophyllus, Artocarpus integer and Mangiferae sp., and fruit vegetables such as Cucumis sativa, Momordica charantia, Luffa sp. and Capsicum sp. (S1). As trapping moved along the highway from Phase 3 to Phase 5 (Lachau to Bintulu area), where secondary jungle and oil palm plantations were found, the number of fruit fly caught dropped dramatically (Table 2).

The main species of fruit fly caught in MElure trap were $B$. papayae $(97.94 \%), B$. umbrosa $(1.50 \%)$ and B. carambolae $(0.49 \%)$ (Table 3). CUE-lure trap has attracted $B$. papayae $(46.00 \%)$, B. caudata $(28.72 \%)$, B. cucurbitae (12.70\%), B. albistrigata (4.62\%), $B$. nigrotibialis $(1.99 \%), \quad B$. carambolae (1.69\%), B. tau (1.42\%) and others $(2.86 \%)$. The quarantine species, $B$. correcta, $B$. dorsalis, $B$. occipitalis and $B$. zonata were absent in both ME-lure and CUE-lure traps. Trimed-lure trap did not detect any Mediteranean fruit fly but had attracted some other species. They were $B$. papayae (41.03\%), B. caudata (19.87\%), B. cucurbitae $(16.67 \%)$, B. albistrigata $(5.13 \%)$ and other species $(17.31 \%)$. 
The geographical distribution patterns of the indigenous fruit fly along the main road of Sarawak are shown in Table 2. All the fruit fly species showed some fluctuations in population as trapping process moved along the main road of Sarawak. Bactrocera papayae and B. caudata were found abundant throughout the trapping locations and $B$. papayae is the predominant species from Phase 1 to Phase 7 (Table 2). This suggests that either $B$. papayae has a wide host range or that its host plant is widely spread throughout Sarawak. The other species were more prevalent at some locations. The population fluctuation patterns were almost similar except for B. apicalis. Generally, the number of fruit fly species trapped declined at secondary jungle areas whereas the number of $B$. apicalis increased in secondary jungle areas. This is because forest fruits served as the host plant for this species (Rohani Ibrahim, Faculty of Agriculture, Universiti Putra Malaysia, personal communications). Out of the eleven identified species, ten of them were detected in all the trapping phases. Dacus hochii was detected in Phase 1, trap no.1 in Bau (S1). Other species which were trapped in low numbers i.e. less than 100 per phase were $B$. apicalis and D. longicornis.

Bactrocera apicalis, D. longicornis and D. hochii are not of economic importance. Bactrocera apicalis, formerly known as $B$. modica (Allwood et al. 1999) is distributed in China (Yunnan, Hainan), Thailand, Vietnam, Phillipines (Mindanao), Malaysia, Brunei and Indonesia. According to Allwood et al. (1999), the flower Trichosanthes wawraei belonging to the Family Cucurbitaceae is the host plant for B. apicalis.

Table 2. Number of fruit fly species caught for 3 types of lures in each phase.

\begin{tabular}{|c|c|c|c|c|c|c|c|c|}
\hline Species/Phase & 1 & 2 & 3 & 4 & 5 & 6 & 7 & Total \\
\hline B. papayae & 44213 & 21016 & 11252 & 16373 & 6933 & 21020 & 30408 & 151215 \\
\hline B. caudata & 1726 & 4574 & 1898 & 738 & 1548 & 1178 & 854 & 12516 \\
\hline B. cucurbitae & 3024 & 1062 & 476 & 207 & 245 & 95 & 437 & 5546 \\
\hline B. umbrosa & 416 & 382 & 226 & 150 & 367 & 134 & 328 & 2003 \\
\hline B. carambolae & 253 & 35 & 94 & 230 & 80 & 237 & 468 & 1397 \\
\hline B. apicalis & 2 & 1 & 27 & 114 & 3 & 11 & 24 & 191 \\
\hline D. longicornis & 13 & 8 & 12 & 5 & 2 & 14 & 2 & 56 \\
\hline D. hochii & 1 & - & - & - & - & - & - & 1 \\
\hline Bactrocera complex species & 156 & 148 & 257 & 233 & 47 & 63 & 145 & 1049 \\
\hline Total & 50638 & 27847 & 14374 & 18138 & 9635 & 23323 & 33541 & 177505 \\
\hline
\end{tabular}

Table 3. Number of fruit fly species caught for 3 types of lures.

\begin{tabular}{|c|c|c|c|c|c|c|}
\hline Species/Lure & ME & $\begin{array}{c}\text { Incidence } \\
(\%)\end{array}$ & CUE & $\begin{array}{c}\text { Incidence } \\
(\%)\end{array}$ & Trimed & $\begin{array}{c}\text { Incidence } \\
(\%)\end{array}$ \\
\hline B. papayae & 131187 & 97.94 & 19964 & 46.00 & 64 & 41.03 \\
\hline B. caudata & 20 & 0.02 & 12465 & 28.72 & 31 & 19.87 \\
\hline B. cucurbitae & 7 & 0.005 & 5513 & 12.70 & 26 & 16.67 \\
\hline B. albistrigata & 27 & 0.02 & 2006 & 4.62 & 8 & 5.13 \\
\hline B. umbrosa & 2002 & 1.50 & - & - & 1 & 0.64 \\
\hline B. carambolae & 662 & 0.49 & 734 & 1.69 & 1 & 0.64 \\
\hline B. nigrotibialis & 1 & 0.001 & 864 & 1.99 & 1 & 0.64 \\
\hline B. tau & 1 & 0.001 & 614 & 1.42 & 9 & 5.77 \\
\hline B. apicalis & - & - & 191 & 0.44 & - & - \\
\hline D. longicornis & - & - & 56 & 0.13 & - & - \\
\hline D. hochii & - & - & 1 & 0.002 & - & - \\
\hline Bactrocera complex species & 40 & 0.03 & 994 & 2.29 & 15 & 9.62 \\
\hline
\end{tabular}

Key: "-" indicates no data. 
Another minor pest, D. longicornis, has been regularly misidentified with numerous synonyms including $D$. semieroides, $D$. destillatoria, $D$. eumenoides and $D$. variegate (Drew et al. 2007). This species attacks plants belonging to the Family Cucurbitaceae such as Luffa acutangula, Luffa aegyptiacca, Melothria wallichii and Trichosanthes cucumerina (Allwood et al. 1999). It is widely spread across the region from Southern Asia to Southeast Asia (Drew et al. 2007).

The GPS data and mapping are important as they provide valuable information such as the exact location where the fruit fly species trapped and the geographical distribution patterns of the fruit fly along the east-west trunk road of Sarawak (S1). This is particularly important when the quarantine species are detected.

The availability of cultivated crops could be considered as the most important environmental factor affecting the population fluctuations. Climatic factors such as rainfall and relative humidity may also influence the population pattern. The unknown species were categorized under Bactrocera complex species due to the difficulty to distinguish them morphologically. Thus, it is suggested that the molecular genetics technique should be adopted for precise identification.

In this survey, the common Solanaceae fruit fly species, B. latifrons was not detected. This species is not attracted to ME or CUE male lures (McQuate 2008). Therefore, a host fruit sampling is needed to confirm the presence or absence of fruit fly species particularly those not attracted to male lures.

\section{CONCLUSION}

There is an abundance of fruit flies along the east-west trunk road of Sarawak. Twenty Dacinae fruit fly species have been trapped and eleven of them were identified as $B$. papayae, B. caudata, B. carambolae, $B$. cucurbitae, $B$. tau, $B$. albistrigata, $B$. nigrotibialis, $B$. umbrosa, B. apicalis, $D$. longicornis and $D$. hochii. Bactrocera papayae, which either has a wide host range or that its host plant is widely spread throughout Sarawak, is the predominant species. The population fluctuations are affected by the types of vegetation. The survey did not detect any foreign quarantine species. This fruit fly surveillance report, reporting the status of fruit fly species in Sarawak, therefore serves as a pest free assurance to the importing and exporting countries based on their quarantine regulations and requirements.

\section{ACKNOWLEDGEMENTS}

This project was funded by Crop Protection Development fund. The assistance rendered by staff from Crop Protection and Quarantine (CPQ), Department of Agriculture (DOA) Sarawak in the trapping exercise is appreciated. The authors thank especially Prof. Madya Dr. Rohani Ibrahim and Dr. Nur Azura Adam from Universiti Putra Malaysia Serdang, Mohd Fakhruddin from CPQ and Mark Ambun from the Entomology Section for their invaluable assistance in the identification process. The authors also thank AAO Ling Kuok Chui from Soil Management Branch, DOA Sarawak for his assistance in producing the map.

\section{REFERENCES}

Allwood, A.J., Chinajariyanwong, A., Kritsaneepaiboon, S., Drew, R.A.I., Hamacek, E.L., Hancock, D.L., Hengsawed, C., Jipanin, J.C., Jirasurat, M., Kong Krong, C., Leong, C. T.S., \& Sijaysegaran, S. (1999). Host plant records for fruit flies (Diptera: Tephritidae) in Southeast Asia. The Raffles Bulletin of Zoology, Supplement No. 7: 1-92.

Drew, R.A.I., Romig, M.C., \& Dorji, C. (2007). Records of Dacinae fruit flies and new species of Dacus (Diptera: Tephritidae) in Bhutan. The Raffles Bulletin of Zoology, 55(1): 1-21.

Hardy, D.E. (1997). The fruit flies (Tephritidae: Diptera) of Thailand and bordering countries. Pacific Insects Monograph 31, 353 pp.

International Plant Protection Convention. (1997). International Standards for Phytosanitary Measures No.6: Guidelines for surveillance. FAO 2006. 4 pp. 
John, L.C. (2008). Encyclopedia of entomology: Traps for capturing insects. Kluwer Academic Publishers. 4346 pp.

McMaugh, T. (2005). Guidelines for surveillance for plant pests in Asia and the Pacific. ACIAR Monograph. 198 pp.

McQuate, G.T. (2008). Age response of male Bactrocera latifrons (Diptera: Tephritidae) to -ionol + cade oil relative to age of sexual maturity. Retrieved from http://www.ars.usda.gov/research/publicati ons.htm on 30th June 2011.

Tan, Y.L. (2004). Identification of Bactrocera genus (Diptera: Tephritidae) via molecular maker. Masters Thesis. Degree of Master of Science. 73 pp.
Vijaysegaran, S. \& Mohd Shamsudin, O. (1991). Fruit flies in Peninsular Malaysia. Their economic importance and control strategies. In K. Kawasaki, O. Iwahashi \& K.Y. Kaneshiro (Eds). Proceeding International Symposium on the Biology and Control of Fruit Flies. Okinawa, Japan, 2-4 September 1991, 105-115.Waterhouse, D.F. (1997). The major invertebrate pests and weeds of agriculture and plantation forestry in the Southern and Western Pacific. ACIAR, Canberra. 93 pp.

White, I.M. \& Elson-Harris, M. (1992). Fruit fly of economic significance: their identification and bionomics. ACIAR and CABI. 601 pp.

\section{Supplementary Materials}

S1. Fruit fly species trapped and vegetation types at each location.

\begin{tabular}{|c|c|c|c|c|c|c|c|c|c|c|c|c|c|c|}
\hline$\frac{\mathscr{D}}{\tilde{a}}$ & 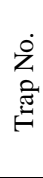 & GPS data & Vegetation Types & 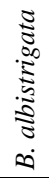 & $\begin{array}{l}: \tilde{\Xi} \\
\tilde{3} \\
\vdots \\
0\end{array}$ & 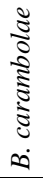 & 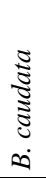 & 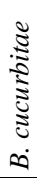 & 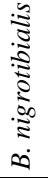 & 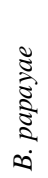 & $\underset{\infty}{3}$ & 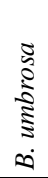 & 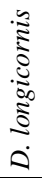 & 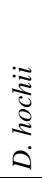 \\
\hline \multirow[t]{15}{*}{1} & 1 & $\begin{array}{l}\mathrm{N} 01^{\circ} 25^{\prime} 34.1^{\prime \prime} \\
\mathrm{E} 110^{\circ} 08^{\prime} 39.6^{\prime \prime}\end{array}$ & $\begin{array}{l}\text { Artocarpus integer, Mangiferae } \mathrm{sp} . \\
\text { Psidium sp., Archidendron pauciflorum }\end{array}$ & $\sqrt{ }$ & & $\sqrt{ }$ & $\sqrt{ }$ & $\sqrt{ }$ & $\sqrt{ }$ & $\sqrt{ }$ & $\sqrt{ }$ & $\sqrt{ }$ & $\sqrt{ }$ & $\sqrt{ }$ \\
\hline & 2 & $\begin{array}{l}\text { N01 }{ }^{\circ} 27^{\prime} 48.0^{\prime \prime} \\
\text { E110 } 07^{\prime} 03.1^{\prime \prime}\end{array}$ & $\begin{array}{l}\text { Musa sp., Durio sp., Mangiferae sp., } \\
\text { Citrus maxima, Nephelium lappaceum, } \\
\text { Syzygium samarangense }\end{array}$ & & & $\sqrt{ }$ & $\sqrt{ }$ & & & $\sqrt{ }$ & $\sqrt{ }$ & $\sqrt{ }$ & & \\
\hline & 3 & $\begin{array}{l}\mathrm{N} 01^{\circ} 29^{\prime} 08.3^{\prime \prime} \\
\mathrm{E} 110^{\circ} 05^{\prime} 08.0^{\prime \prime}\end{array}$ & $\begin{array}{l}\text { Musa sp., Artocarpus integer, Citrus } \\
\text { maxima, Nephelium lappaceum }\end{array}$ & & & $\sqrt{ }$ & $\sqrt{ }$ & $\sqrt{ }$ & $\sqrt{ }$ & $\sqrt{ }$ & $\sqrt{ }$ & $\sqrt{ }$ & $\sqrt{ }$ & \\
\hline & 4 & $\begin{array}{l}\mathrm{N}^{\circ} 1^{\circ} 29^{\prime} 23.8^{\prime \prime} \\
\mathrm{E} 110^{\circ} 02^{\prime} 31.6^{\prime \prime}\end{array}$ & $\begin{array}{l}\text { Durio sp., Lansium domesticum, } \\
\text { Mangiferae sp., Artocarpus } \\
\text { heterophyllus, Citrus maxima }\end{array}$ & $\sqrt{ }$ & & $\sqrt{ }$ & $\sqrt{ }$ & $\sqrt{ }$ & $\sqrt{ }$ & $\sqrt{ }$ & $\sqrt{ }$ & $\sqrt{ }$ & & \\
\hline & 5 & $\begin{array}{l}\text { N01 }{ }^{\circ} 28^{\prime} 41.4^{\prime \prime} \\
\text { E109 } 59^{\prime} 45.2^{\prime \prime}\end{array}$ & $\begin{array}{l}\text { Psidium sp., Lansium domesticum, Litsea } \\
\text { garciae, mata kucing, Mangiferae sp., } \\
\text { Artocarpus heterophyllus, Averrhoa } \\
\text { carambola, Nephelium lappaceum }\end{array}$ & & & $\sqrt{ }$ & $\sqrt{ }$ & $\sqrt{ }$ & $\sqrt{ }$ & $\sqrt{ }$ & & $\sqrt{ }$ & & \\
\hline & 6 & $\begin{array}{l}\text { N01 }{ }^{\circ} 28^{\prime} 58.5^{\prime \prime} \\
\text { E109 } 57^{\prime} 18.9^{\prime \prime}\end{array}$ & $\begin{array}{l}\text { Artocarpus integer, Citrus sp., Psidium } \\
\text { sp., Syzygium samarangense, Nephelium } \\
\text { lappaceum, Piper nigrum, Capsicum sp. }\end{array}$ & & & $\sqrt{ }$ & $\sqrt{ }$ & $\sqrt{ }$ & $\sqrt{ }$ & $\sqrt{ }$ & $\sqrt{ }$ & $\sqrt{ }$ & & \\
\hline & 7 & $\begin{array}{l}\text { N0130'31.0" } \\
\text { E109॰54'40.4" }\end{array}$ & Secondary jungle, Acacia & & & $\sqrt{ }$ & $\sqrt{ }$ & $\sqrt{ }$ & $\sqrt{ }$ & $\sqrt{ }$ & & & & \\
\hline & 8 & $\begin{array}{l}\text { N01'33'35.4" } \\
\text { E109 } 53^{\prime} 17.0^{\prime \prime}\end{array}$ & Musa sp., Mangiferae sp., Pangium edule & $\sqrt{ }$ & & $\sqrt{ }$ & $\sqrt{ }$ & $\sqrt{ }$ & $\sqrt{ }$ & $\sqrt{ }$ & $\sqrt{ }$ & $\sqrt{ }$ & & \\
\hline & 9 & $\begin{array}{l}\text { N0136'01.7" } \\
\text { E109॰53'21.8" }\end{array}$ & $\begin{array}{l}\text { Syzygium samarangense, Lansium } \\
\text { domesticum, Capsicum sp. }\end{array}$ & & & & & & $\sqrt{ }$ & $\sqrt{ }$ & & $\sqrt{ }$ & & \\
\hline & 10 & $\begin{array}{l}\text { N0138'35.0" } \\
\text { E109॰52'25.9" }\end{array}$ & $\begin{array}{l}\text { Artocarpus integer, Cocos nucifera, } \\
\text { Mangiferae } \mathrm{sp} .\end{array}$ & $\sqrt{ }$ & & $\sqrt{ }$ & $\sqrt{ }$ & $\sqrt{ }$ & $\sqrt{ }$ & $\sqrt{ }$ & & $\sqrt{ }$ & $\sqrt{ }$ & \\
\hline & 11 & $\begin{array}{l}\text { N01²8'06.3" } \\
\text { E110॰19'00.2" }\end{array}$ & Secondary jungle & & & $\sqrt{ }$ & $\sqrt{ }$ & & & $\sqrt{ }$ & $\sqrt{ }$ & & & \\
\hline & 12 & $\begin{array}{l}\text { N0127'16.1" } \\
\text { E110॰16'51.0" }\end{array}$ & Mangiferae sp. & $\sqrt{ }$ & & $\sqrt{ }$ & $\sqrt{ }$ & $\sqrt{ }$ & $\sqrt{ }$ & $\sqrt{ }$ & $\sqrt{ }$ & $\sqrt{ }$ & & \\
\hline & 13 & $\begin{array}{l}\text { N0126'42.4" } \\
\text { E110॰14'05.4" }\end{array}$ & Nephelium lappaceum & & $\sqrt{ }$ & $\sqrt{ }$ & $\sqrt{ }$ & $\sqrt{ }$ & $\sqrt{ }$ & $\sqrt{ }$ & $\sqrt{ }$ & $\sqrt{ }$ & $\sqrt{ }$ & \\
\hline & 14 & $\begin{array}{l}\mathrm{N}^{\circ} 1^{\circ} 25^{\prime} 49.5^{\prime \prime} \\
\mathrm{E} 110^{\circ} 11^{\prime} 39.0^{\prime \prime}\end{array}$ & Manilkara zapota, Durio sp. belanda & & & $\sqrt{ }$ & $\sqrt{ }$ & & $\sqrt{ }$ & $\sqrt{ }$ & & $\sqrt{ }$ & $\sqrt{ }$ & \\
\hline & 15 & $\begin{array}{l}\text { N01²5'15.6" } \\
\text { E11009'59.6" }\end{array}$ & Nephelium lappaceum & $\sqrt{ }$ & & $\sqrt{ }$ & $\sqrt{ }$ & $\sqrt{ }$ & $\sqrt{ }$ & $\sqrt{ }$ & $\sqrt{ }$ & & & \\
\hline
\end{tabular}




\begin{tabular}{|c|c|c|c|c|c|c|c|c|c|c|c|c|c|c|}
\hline 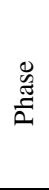 & 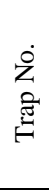 & GPS data & Vegetation Types & 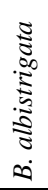 & 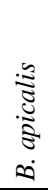 & 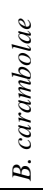 & 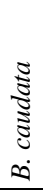 & 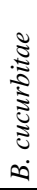 & 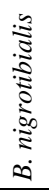 & 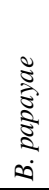 & $\underset{\infty}{\infty}$ & 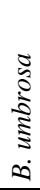 & 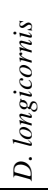 & $\begin{array}{c}: \widetilde{z} \\
\vdots \\
\vdots \\
\vdots \\
0\end{array}$ \\
\hline & 16 & $\begin{array}{l}\text { N01 }{ }^{\circ} 39^{\prime} 13.5^{\prime \prime} \\
\text { E109 } 50^{\prime} 07.6^{\prime \prime}\end{array}$ & Secondary jungle & $\sqrt{ }$ & & & $\sqrt{ }$ & & $\sqrt{ }$ & $\sqrt{ }$ & & & $\sqrt{ }$ & \\
\hline & 17 & $\begin{array}{l}\text { N01 } 39^{\prime} 43.9^{\prime \prime} \\
\text { E10948'10.3" }\end{array}$ & Dimocarpus longan & $\sqrt{ }$ & & $\sqrt{ }$ & $\sqrt{ }$ & $\sqrt{ }$ & $\sqrt{ }$ & $\sqrt{ }$ & & $\sqrt{ }$ & & \\
\hline & 18 & $\begin{array}{l}\text { N014' } 47.5^{\prime \prime} \\
\text { E109॰46'53.3" }\end{array}$ & $\begin{array}{l}\text { Musa sp., Citrus sp. sp., Lansium } \\
\text { domesticum, Mangiferae sp., Mangiferae } \\
\text { sp.steen, Carica Carica papaya, } \\
\text { Nephelium lappaceum, Syzygium } \\
\text { samarangense }\end{array}$ & & & & & & & & & & & \\
\hline & 19 & $\begin{array}{l}\text { N01 }{ }^{\circ} 43^{\prime} 56.4^{\prime \prime} \\
\text { E10946'03.6" }\end{array}$ & $\begin{array}{l}\text { Musa sp., Psidium sp., Syzygium } \\
\text { samarangense, Momordica charantia, } \\
\text { Capsicum sp., Vigna unguiculata } \\
\text { sesquipedalis, Cucumis sativa }\end{array}$ & $\sqrt{ }$ & & & $\sqrt{ }$ & $\sqrt{ }$ & $\sqrt{ }$ & $\sqrt{ }$ & $\sqrt{ }$ & $\sqrt{ }$ & & \\
\hline & 20 & $\begin{array}{l}\text { N01 }{ }^{\circ} 46^{\prime} 05.2^{\prime \prime} \\
\text { E10944'48.1" }\end{array}$ & Nephelium lappaceum & $\sqrt{ }$ & & $\sqrt{ }$ & $\sqrt{ }$ & $\sqrt{ }$ & & $\sqrt{ }$ & & & & \\
\hline & 21 & $\begin{array}{l}\text { N01 }{ }^{\circ} 47^{\prime} 56.9^{\prime \prime} \\
\text { E1094 } 45^{\prime} 11.3^{\prime \prime}\end{array}$ & Mangiferae sp. & $\sqrt{ }$ & & $\sqrt{ }$ & $\sqrt{ }$ & $\sqrt{ }$ & $\sqrt{ }$ & $\sqrt{ }$ & $\sqrt{ }$ & $\sqrt{ }$ & $\sqrt{ }$ & \\
\hline & 22 & $\begin{array}{l}\text { N01 }{ }^{\circ} 38^{\prime} 02.5^{\prime \prime} \\
\text { E109} 46^{\prime} 17.6^{\prime \prime}\end{array}$ & $\begin{array}{l}\text { Asam, Artocarpus integer, Annona } \\
\text { muricata, Mangiferae sp., Artocarpus } \\
\text { heterophyllus, Nephelium lappaceum, } \\
\text { Capsicum sp. }\end{array}$ & & & $\sqrt{ }$ & $\sqrt{ }$ & $\sqrt{ }$ & $\sqrt{ }$ & $\sqrt{ }$ & $\sqrt{ }$ & $\sqrt{ }$ & & \\
\hline & 23 & $\begin{array}{l}\mathrm{N}^{\circ} 1^{\circ} 38^{\prime} 21.1^{\prime \prime} \\
\mathrm{E} 109^{\circ} 43^{\prime} 57.2^{\prime \prime}\end{array}$ & $\begin{array}{l}\text { Artocarpus integer, Dimocarpus longan, } \\
\text { Artocarpus heterophyllus, Carica } \\
\text { papaya, Averrhoa carambola }\end{array}$ & $\sqrt{ }$ & & $\sqrt{ }$ & $\sqrt{ }$ & $\sqrt{ }$ & $\sqrt{ }$ & $\sqrt{ }$ & $\sqrt{ }$ & $\sqrt{ }$ & & \\
\hline & 24 & $\begin{array}{l}\text { N01 } 1^{\circ} 38^{\prime} 26.9^{\prime \prime} \\
\text { E109 } 41^{\prime} 43.3^{\prime \prime}\end{array}$ & $\begin{array}{l}\text { Musa sp., Mangiferae sp., Nephelium } \\
\text { lappaceum }\end{array}$ & & & $\sqrt{ }$ & $\sqrt{ }$ & $\sqrt{ }$ & $\sqrt{ }$ & $\sqrt{ }$ & $\sqrt{ }$ & $\sqrt{ }$ & & \\
\hline & 25 & 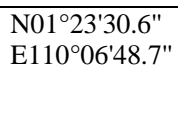 & $\begin{array}{l}\text { Citrus sp., Artocarpus integer, } \\
\text { Dimocarpus longan, Mangiferae sp., } \\
\text { Nephelium lappaceum, Syzygium } \\
\text { samarangense }\end{array}$ & & & $\sqrt{ }$ & $\sqrt{ }$ & & $\sqrt{ }$ & $\sqrt{ }$ & $\sqrt{ }$ & & & \\
\hline & 26 & $\begin{array}{l}\text { N01 }{ }^{\circ} 21^{\prime} 57.3^{\prime \prime} \\
\text { E11004'55.5" }\end{array}$ & $\begin{array}{l}\text { Theobroma cocoa, Citrus sp., Capsicum } \\
\text { sp. }\end{array}$ & & & $\sqrt{ }$ & $\sqrt{ }$ & $\sqrt{ }$ & $\sqrt{ }$ & $\sqrt{ }$ & $\sqrt{ }$ & & $\sqrt{ }$ & \\
\hline & 27 & $\begin{array}{l}\mathrm{N}^{\circ} 1^{\circ} 20^{\prime} 40.3^{\prime \prime} \\
\mathrm{E} 110^{\circ} 02^{\prime} 51.3^{\prime \prime}\end{array}$ & Musa sp., Artocarpus integer & & & & $\sqrt{ }$ & & $\sqrt{ }$ & $\sqrt{ }$ & & $\sqrt{ }$ & & \\
\hline & 28 & $\begin{array}{l}\mathrm{N} 01^{\circ} 20^{\prime} 02.2^{\prime \prime} \\
\mathrm{E} 110^{\circ} 00^{\prime} 42.5^{\prime \prime}\end{array}$ & Citrus sp. & & & & $\sqrt{ }$ & $\sqrt{ }$ & $\sqrt{ }$ & $\sqrt{ }$ & & $\sqrt{ }$ & $\sqrt{ }$ & \\
\hline \multirow[t]{12}{*}{2} & 1 & $\begin{array}{l}\text { N01 } 08^{\prime} 46.6^{\prime \prime} \\
\text { E110³1'49.5" }\end{array}$ & Nephelium lappaceum & & & & $\sqrt{ }$ & $\sqrt{ }$ & $\sqrt{ }$ & $\sqrt{ }$ & & & & \\
\hline & 2 & $\begin{array}{l}\text { N01 }{ }^{\circ} 06^{\prime} 49.2^{\prime \prime} \\
\text { E110³0'51.7" }\end{array}$ & Secondary jungle & $\sqrt{ }$ & & $\sqrt{ }$ & $\sqrt{ }$ & $\sqrt{ }$ & $\sqrt{ }$ & $\sqrt{ }$ & $\sqrt{ }$ & $\sqrt{ }$ & & \\
\hline & 3 & $\begin{array}{l}\text { N01 }{ }^{\circ} 06^{\prime} 28.8^{\prime \prime} \\
\mathrm{E} 110^{\circ} 28^{\prime} 21.5^{\prime \prime}\end{array}$ & Secondary jungle & & & & $\sqrt{ }$ & $\sqrt{ }$ & $\sqrt{ }$ & $\sqrt{ }$ & & & & \\
\hline & 4 & $\begin{array}{l}\text { N01 }{ }^{\circ} 07^{\prime} 44.5^{\prime \prime} \\
\text { E1 } 10^{\circ} 25^{\prime} 24.2^{\prime \prime}\end{array}$ & Secondary jungle & & & & $\sqrt{ }$ & & $\sqrt{ }$ & $\sqrt{ }$ & $\sqrt{ }$ & $\sqrt{ }$ & & \\
\hline & 5 & $\begin{array}{l}\text { N01 }{ }^{\circ} 06^{\prime} 25.1^{\prime \prime} \\
\text { E11023'27.0" }\end{array}$ & Secondary jungle & & & & & & $\sqrt{ }$ & $\sqrt{ }$ & & & & \\
\hline & 6 & 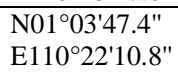 & Secondary jungle & $\sqrt{ }$ & $\sqrt{ }$ & & $\sqrt{ }$ & & $\sqrt{ }$ & $\sqrt{ }$ & $\sqrt{ }$ & $\sqrt{ }$ & & \\
\hline & 7 & $\begin{array}{l}\text { N01 }{ }^{\circ} 01^{\prime} 36.8^{\prime \prime} \\
\mathrm{E} 110^{\circ} 21^{\prime} 26.1^{\prime \prime}\end{array}$ & Secondary jungle & $\sqrt{ }$ & & & $\sqrt{ }$ & $\sqrt{ }$ & & $\sqrt{ }$ & $\sqrt{ }$ & $\sqrt{ }$ & & \\
\hline & 8 & $\begin{array}{l}\text { N0059'30.7" } \\
\text { E110²0'55.1" }\end{array}$ & Secondary jungle & $\sqrt{ }$ & & $\sqrt{ }$ & $\sqrt{ }$ & & $\sqrt{ }$ & $\sqrt{ }$ & $\sqrt{ }$ & & & \\
\hline & 9 & $\begin{array}{l}\text { N01 }{ }^{\circ} 10^{\prime} 53.3^{\prime \prime} \\
\text { E110³3'37. }\end{array}$ & $\begin{array}{l}\text { Musa sp., Nephelium lappaceum, } \\
\text { Saurapus androgynous, Capsicum sp., } \\
\text { Luffa sp., Cucumis sativa }\end{array}$ & $\sqrt{ }$ & & $\sqrt{ }$ & $\sqrt{ }$ & $\sqrt{ }$ & $\sqrt{ }$ & $\sqrt{ }$ & $\sqrt{ }$ & $\sqrt{ }$ & & \\
\hline & 10 & $\begin{array}{l}\text { N01 }{ }^{\circ} 12^{\prime} 25.1^{\prime \prime} \\
\text { E110³1'33.9" }\end{array}$ & $\begin{array}{l}\text { Artocarpus heterophyllus, nangcem, } \\
\text { Carica papaya, Averrhoa carambola, } \\
\text { Vigna unguiculata sesquipedalis, } \\
\text { Cucumis sativa, Momordica charantia, }\end{array}$ & $\sqrt{ }$ & & $\sqrt{ }$ & $\sqrt{ }$ & $\sqrt{ }$ & $\sqrt{ }$ & $\sqrt{ }$ & $\sqrt{ }$ & $\sqrt{ }$ & & \\
\hline & 11 & $\begin{array}{l}\mathrm{N}^{\circ} 1^{\circ} 14^{\prime} 37.5^{\prime \prime} \\
\mathrm{E} 110^{\circ} 29^{\prime} 22.3^{\prime \prime}\end{array}$ & $\begin{array}{l}\text { Musa sp., Artocarpus integer, Syzygium } \\
\text { samarangense }\end{array}$ & & & & $\sqrt{ }$ & $\sqrt{ }$ & $\sqrt{ }$ & $\sqrt{ }$ & $\sqrt{ }$ & $\sqrt{ }$ & & \\
\hline & 12 & $\begin{array}{l}\text { N01 }{ }^{\circ} 16^{\prime} 22.7^{\prime \prime} \\
\mathrm{E} 110^{\circ} 27^{\prime} 12.2^{\prime \prime}\end{array}$ & $\begin{array}{l}\text { Musa sp., Citrus sp., Manilkara zapota, } \\
\text { Solanum melongena, Artocarpus integer, } \\
\text { Syzygium samarangense, Momordica } \\
\text { charantia, Capsicum sp., Cucumis sativa }\end{array}$ & $\sqrt{ }$ & & & $\sqrt{ }$ & $\sqrt{ }$ & $\sqrt{ }$ & $\sqrt{ }$ & $\sqrt{ }$ & $\sqrt{ }$ & & \\
\hline
\end{tabular}




\begin{tabular}{|c|c|c|c|c|c|c|c|c|c|c|c|c|c|c|}
\hline 离 & $\begin{array}{l}\dot{z} \\
\text { 吾 } \\
\text { H }\end{array}$ & GPS data & Vegetation Types & 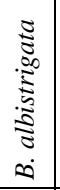 & 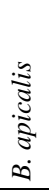 & 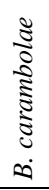 & 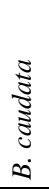 & 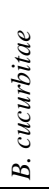 & 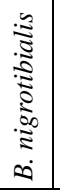 & $\begin{array}{c}\tilde{\Xi} \\
\vdots \\
\vdots \\
\vdots \\
\vdots \\
\infty\end{array}$ & 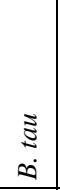 & 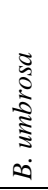 & 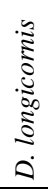 & $\begin{array}{l}: \widetilde{3} \\
\vdots \\
\vdots \\
\vdots \\
0\end{array}$ \\
\hline & 13 & $\begin{array}{l}\text { N01 } 17^{\prime} 19.3^{\prime \prime} \\
\text { E110²4'54.0" }\end{array}$ & $\begin{array}{l}\text { Musa sp., Citrus sp., Citrus maxima, } \\
\text { Carica papaya, leafy vegetables }\end{array}$ & & & & $\sqrt{ }$ & $\sqrt{ }$ & $\sqrt{ }$ & $\sqrt{ }$ & $\sqrt{ }$ & $\sqrt{ }$ & & \\
\hline & 14 & $\begin{array}{l}\text { N01 }{ }^{\circ} 19^{\prime} 36.4^{\prime \prime} \\
\text { E110²4'11.3" }\end{array}$ & Secondary jungle & & & & $\sqrt{ }$ & $\sqrt{ }$ & $\sqrt{ }$ & $\sqrt{ }$ & $\sqrt{ }$ & $\sqrt{ }$ & & \\
\hline & 15 & $\begin{array}{l}\mathrm{N}^{\circ} 1^{\circ} 22^{\prime} 18.7^{\prime \prime} \\
\mathrm{E} 110^{\circ} 23^{\prime} 14.2^{\prime \prime}\end{array}$ & Musa sp., Citrus sp., Capsicum sp. & $\sqrt{ }$ & & & $\sqrt{ }$ & $\sqrt{ }$ & $\sqrt{ }$ & $\sqrt{ }$ & $\sqrt{ }$ & $\sqrt{ }$ & & \\
\hline & 16 & $\begin{array}{l}\text { N01 } 08^{\prime} 56.4^{\prime \prime} \\
\text { E110 } 34^{\prime} 43.6^{\prime \prime}\end{array}$ & Citrus sp. & $\sqrt{ }$ & & & $\sqrt{ }$ & $\sqrt{ }$ & $\sqrt{ }$ & $\sqrt{ }$ & $\sqrt{ }$ & $\sqrt{ }$ & $\sqrt{ }$ & \\
\hline & 17 & $\begin{array}{l}\text { N01 } 07^{\prime} 32.3^{\prime \prime} \\
\mathrm{E} 110^{\circ} 37^{\prime} 09.1^{\prime \prime}\end{array}$ & Citrus sp. & $\sqrt{ }$ & & $\sqrt{ }$ & $\sqrt{ }$ & $\sqrt{ }$ & & $\sqrt{ }$ & & $\sqrt{ }$ & & \\
\hline & 18 & $\begin{array}{l}\text { N01 } 05^{\prime} 17.2^{\prime \prime} \\
\text { E110 } 37^{\prime} 46.7^{\prime \prime}\end{array}$ & Secondary jungle & & & & $\sqrt{ }$ & $\sqrt{ }$ & $\sqrt{ }$ & $\sqrt{ }$ & $\sqrt{ }$ & $\sqrt{ }$ & $\sqrt{ }$ & \\
\hline & 19 & $\begin{array}{l}\text { N01 } 03 ' 17.4^{\prime \prime} \\
\text { E110³9'14.9" }\end{array}$ & Citrus sp. sp. & & & & $\sqrt{ }$ & $\sqrt{ }$ & & $\sqrt{ }$ & & $\sqrt{ }$ & & \\
\hline & 20 & $\begin{array}{l}\text { N01 } 02^{\prime} 23.1^{\prime \prime} \\
\mathrm{E} 110^{\circ} 41^{\prime} 47.4^{\prime \prime}\end{array}$ & Secondary jungle & & & $\sqrt{ }$ & $\sqrt{ }$ & $\sqrt{ }$ & $\sqrt{ }$ & $\sqrt{ }$ & & $\sqrt{ }$ & & \\
\hline & 21 & $\begin{array}{l}\text { N01 } 02^{\prime} 24.8^{\prime \prime} \\
\mathrm{E} 110^{\circ} 44^{\prime} 12.0^{\prime \prime}\end{array}$ & Syzygium samarangense & & & $\sqrt{ }$ & $\sqrt{ }$ & $\sqrt{ }$ & & $\sqrt{ }$ & $\sqrt{ }$ & & & \\
\hline & 22 & $\begin{array}{l}\mathrm{N} 01^{\circ} 02^{\prime} 29.7^{\prime \prime} \\
\mathrm{E} 110^{\circ} 46^{\prime} 22.1^{\prime \prime}\end{array}$ & Secondary jungle & & & $\sqrt{ }$ & $\sqrt{ }$ & $\sqrt{ }$ & $\sqrt{ }$ & $\sqrt{ }$ & & & & \\
\hline & 23 & $\begin{array}{l}\mathrm{N} 01^{\circ} 02^{\prime} 31.2^{\prime \prime} \\
\mathrm{E} 110^{\circ} 49^{\prime} 29.1^{\prime \prime}\end{array}$ & Citrus sp. & & & & $\sqrt{ }$ & & & $\sqrt{ }$ & & & & \\
\hline & 24 & $\begin{array}{l}\text { N01 } 03 ' 28.9^{\prime \prime} \\
\text { E110 } 52 ' 21.1^{\prime \prime}\end{array}$ & Secondary jungle & & & & $\sqrt{ }$ & $\sqrt{ }$ & $\sqrt{ }$ & $\sqrt{ }$ & $\sqrt{ }$ & $\sqrt{ }$ & $\sqrt{ }$ & \\
\hline & 25 & $\begin{array}{l}\text { N01 } 04^{\prime} 21.9^{\prime \prime} \\
\text { E110 } 544^{\prime} 54.2^{\prime \prime}\end{array}$ & Secondary jungle & & & & $\sqrt{ }$ & & $\sqrt{ }$ & $\sqrt{ }$ & & & & \\
\hline & 26 & $\begin{array}{l}\text { N01 } 04^{\prime} 33.7^{\prime \prime} \\
\text { E110 } 58^{\prime} 18.0^{\prime \prime}\end{array}$ & Nephelium lappaceum & & & & & & $\sqrt{ }$ & $\sqrt{ }$ & & $\sqrt{ }$ & & \\
\hline & 27 & $\begin{array}{l}\text { N01 } 04^{\prime} 39.8^{\prime \prime} \\
\mathrm{E} 110^{\circ} 01^{\prime} 30.8^{\prime \prime}\end{array}$ & Secondary jungle & & & & $\sqrt{ }$ & & & $\sqrt{ }$ & & & & \\
\hline & 28 & $\begin{array}{l}\text { N01 } 04^{\prime} 50.7^{\prime \prime} \\
\text { E11004'01.1" }\end{array}$ & Secondary jungle & & & $\sqrt{ }$ & $\sqrt{ }$ & $\sqrt{ }$ & $\sqrt{ }$ & $\sqrt{ }$ & & & & \\
\hline & 29 & $\begin{array}{l}\text { N01 } 04^{\prime} 44.7^{\prime \prime} \\
\text { E110 } 07^{\prime} 24.8^{\prime \prime}\end{array}$ & Secondary jungle & & & & $\sqrt{ }$ & $\sqrt{ }$ & $\sqrt{ }$ & $\sqrt{ }$ & & & & \\
\hline & 30 & $\begin{array}{l}\text { N01 } 04^{\prime} 30.9^{\prime \prime} \\
\text { E11009'18.1" }\end{array}$ & Secondary jungle & & & & $\sqrt{ }$ & & $\sqrt{ }$ & $\sqrt{ }$ & & & & \\
\hline \multirow[t]{14}{*}{3} & 1 & $\begin{array}{l}\text { N01 } 05^{\prime} 30.4^{\prime \prime} \\
\mathrm{E} 111^{\circ} 11^{\prime} 52.5^{\prime \prime}\end{array}$ & Secondary jungle & & $\sqrt{ }$ & $\sqrt{ }$ & $\sqrt{ }$ & & $\sqrt{ }$ & $\sqrt{ }$ & $\sqrt{ }$ & & & \\
\hline & 2 & $\begin{array}{l}\text { N01 } 05^{\circ} 54.4^{\prime \prime} \\
\text { E111 }{ }^{\circ} 13 ' 36.6^{\prime \prime}\end{array}$ & Secondary jungle & & & & & $\sqrt{ }$ & $\sqrt{ }$ & $\sqrt{ }$ & & & & \\
\hline & 3 & $\begin{array}{l}\mathrm{N}^{\circ} 1^{\circ} 05^{\prime} 03.9^{\prime \prime} \\
\mathrm{E} 111^{\circ} 16^{\prime} 02.2^{\prime \prime}\end{array}$ & Secondary jungle & & & $\sqrt{ }$ & $\sqrt{ }$ & & & $\sqrt{ }$ & & & & \\
\hline & 4 & $\begin{array}{l}\text { N01 }{ }^{\circ} 04^{\prime} 03.2^{\prime \prime} \\
\mathrm{E} 111^{\circ} 18^{\prime} 30.1^{\prime \prime}\end{array}$ & Secondary jungle & & & $\sqrt{ }$ & $\sqrt{ }$ & & $\sqrt{ }$ & $\sqrt{ }$ & & & & \\
\hline & 5 & $\begin{array}{l}\mathrm{N} 01^{\circ} 04^{\prime} 10.6^{\prime \prime} \\
\mathrm{E} 111^{\circ} 21^{\prime} 02.9^{\prime \prime}\end{array}$ & Secondary jungle & & & & $\sqrt{ }$ & & & $\sqrt{ }$ & & $\sqrt{ }$ & & \\
\hline & 6 & $\begin{array}{l}\mathrm{N} 01^{\circ} 05^{\prime} 38.7^{\prime \prime} \\
\mathrm{E} 111^{\circ} 23^{\prime} 01.2^{\prime \prime}\end{array}$ & Secondary jungle & & & & $\sqrt{ }$ & $\sqrt{ }$ & $\sqrt{ }$ & $\sqrt{ }$ & $\sqrt{ }$ & $\sqrt{ }$ & & \\
\hline & 7 & $\begin{array}{l}{\mathrm{N} 01^{\circ} 06}^{\circ} 59.8^{\prime \prime} \\
\mathrm{E} 111^{\circ} 24 ' 59.3^{\prime \prime}\end{array}$ & Secondary jungle & $\sqrt{ }$ & $\sqrt{ }$ & $\sqrt{ }$ & $\sqrt{ }$ & $\sqrt{ }$ & $\sqrt{ }$ & $\sqrt{ }$ & $\sqrt{ }$ & $\sqrt{ }$ & & \\
\hline & 8 & $\begin{array}{l}\text { N01 }{ }^{\circ} 09^{\prime} 01.1^{\prime \prime} \\
\mathrm{E} 111^{\circ} 26^{\prime} 25.2^{\prime \prime}\end{array}$ & $\begin{array}{l}\text { Musa sp., Mangiferae sp., Artocarpus } \\
\text { heterophyllus, Averrhoa carambola }\end{array}$ & $\sqrt{ }$ & & $\sqrt{ }$ & $\sqrt{ }$ & $\sqrt{ }$ & & $\sqrt{ }$ & $\sqrt{ }$ & $\sqrt{ }$ & & \\
\hline & 9 & $\begin{array}{l}\mathrm{N}^{\circ} 01^{\circ} 08^{\prime} 54.6^{\prime \prime} \\
\mathrm{E} 111^{\circ} 28^{\prime} 20.5^{\prime \prime}\end{array}$ & Nephelium lappaceum, Capsicum sp. & $\sqrt{ }$ & & & $\sqrt{ }$ & $\sqrt{ }$ & $\sqrt{ }$ & $\sqrt{ }$ & & $\sqrt{ }$ & & \\
\hline & 10 & $\begin{array}{l}\text { N0107'33.7" } \\
\text { E111 } 31^{\circ} 30.1^{\prime \prime}\end{array}$ & Psidium sp. & & & $\sqrt{ }$ & $\sqrt{ }$ & $\sqrt{ }$ & $\sqrt{ }$ & $\sqrt{ }$ & $\sqrt{ }$ & $\sqrt{ }$ & & \\
\hline & 11 & $\begin{array}{l}\text { N01 } 07^{\circ} 17.8^{\prime \prime} \\
\text { E111 } 32 ' 30.7^{\circ}\end{array}$ & Secondary jungle & & & & $\sqrt{ }$ & $\sqrt{ }$ & $\sqrt{ }$ & $\sqrt{ }$ & & $\sqrt{ }$ & & \\
\hline & 12 & 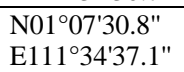 & Secondary jungle & & & & $\sqrt{ }$ & $\sqrt{ }$ & & $\sqrt{ }$ & $\sqrt{ }$ & $\sqrt{ }$ & & \\
\hline & 13 & $\begin{array}{l}\text { N01 }{ }^{\circ} 07^{\prime} 32.4^{\prime \prime} \\
\mathrm{E} 111^{\circ} 37^{\prime} 04.8^{\prime \prime}\end{array}$ & Secondary jungle & & $\sqrt{ }$ & $\sqrt{ }$ & $\sqrt{ }$ & $\sqrt{ }$ & $\sqrt{ }$ & $\sqrt{ }$ & & & & \\
\hline & 14 & $\begin{array}{l}\text { N01 }{ }^{\circ} 09^{\prime} 11.4^{\prime \prime} \\
\text { E111 } 38^{\prime} 58.7^{\prime \prime}\end{array}$ & Secondary jungle & & $\sqrt{ }$ & & $\sqrt{ }$ & $\sqrt{ }$ & & $\sqrt{ }$ & & $\sqrt{ }$ & & \\
\hline
\end{tabular}




\begin{tabular}{|c|c|c|c|c|c|c|c|c|c|c|c|c|c|c|}
\hline $\begin{array}{l}\mathscr{y} \\
\frac{\mathscr{\Xi}}{2}\end{array}$ & 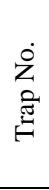 & GPS data & Vegetation Types & 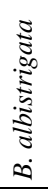 & 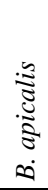 & 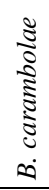 & $\begin{array}{c}\mathbb{3} \\
\mathbb{Z} \\
\mathbb{Z} \\
0 \\
\infty\end{array}$ & 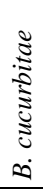 & 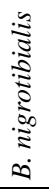 & 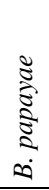 & $\underset{\infty}{\vdots}$ & $\begin{array}{r} \\
\vdots \\
\vdots \\
\vdots \\
\vdots \\
\vdots \\
\infty\end{array}$ & 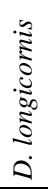 & $\begin{array}{l}: \vdots \\
\vdots \\
\Xi \\
\vdots \\
0\end{array}$ \\
\hline & 15 & $\begin{array}{l}\text { N01 }{ }^{\circ} 12^{\prime} 01.4^{\prime \prime} \\
\mathrm{E}_{111^{\circ} 39^{\prime} 11.8^{\prime \prime}}\end{array}$ & Secondary jungle & & & $\sqrt{ }$ & $\sqrt{ }$ & $\sqrt{ }$ & $\sqrt{ }$ & $\sqrt{ }$ & & $\sqrt{ }$ & & \\
\hline & 16 & $\begin{array}{l}\text { N01 }{ }^{\circ} 14^{\prime} 31.9^{\prime \prime} \\
\text { E111 } 38^{\prime} 33.4^{\prime \prime}\end{array}$ & Secondary jungle & & & & & & & $\sqrt{ }$ & & $\sqrt{ }$ & & \\
\hline & 17 & $\begin{array}{l}\text { N01 }{ }^{\circ} 16^{\prime} 39.2^{\prime \prime} \\
\text { E110³7'29.3" }\end{array}$ & Secondary jungle & & $\sqrt{ }$ & & $\sqrt{ }$ & $\sqrt{ }$ & $\sqrt{ }$ & $\sqrt{ }$ & & $\sqrt{ }$ & & \\
\hline & 18 & $\begin{array}{l}\mathrm{N}^{\circ} 1^{\circ} 19^{\prime} 02.4^{\prime \prime} \\
\mathrm{E} 111^{\circ} 37^{\prime} 06.0^{\prime \prime}\end{array}$ & Secondary jungle, Musa sp. & & & $\sqrt{ }$ & $\sqrt{ }$ & $\sqrt{ }$ & $\sqrt{ }$ & $\sqrt{ }$ & & & & \\
\hline & 19 & $\begin{array}{l}\text { N01²1'10.1" } \\
\text { E111 } 36^{\circ} 20.0^{\prime \prime}\end{array}$ & $\begin{array}{l}\text { Secondary jungle, Musa sp., bamboo, } \\
\text { Mangiferae sp. }\end{array}$ & & $\sqrt{ }$ & & $\sqrt{ }$ & $\sqrt{ }$ & & $\sqrt{ }$ & & $\sqrt{ }$ & & \\
\hline & 20 & $\begin{array}{l}\mathrm{N}^{\circ} 1^{\circ} 23 ' 22.5^{\prime \prime} \\
\mathrm{E} 111^{\circ} 35^{\prime} 52.4^{\prime \prime}\end{array}$ & Secondary jungle & & & $\sqrt{ }$ & $\sqrt{ }$ & $\sqrt{ }$ & $\sqrt{ }$ & $\sqrt{ }$ & & & & \\
\hline & 21 & $\begin{array}{l}\mathrm{N}^{\circ} 1^{\circ} 25^{\prime} 42.3^{\prime \prime} \\
\mathrm{E} 111^{\circ} 34^{\prime} 59.8^{\prime \prime}\end{array}$ & Secondary jungle & & & $\sqrt{ }$ & $\sqrt{ }$ & & & $\sqrt{ }$ & & & & \\
\hline & 22 & $\begin{array}{l}\mathrm{N}^{\circ} 1^{\circ} 27^{\prime} 42.1^{\prime \prime} \\
\mathrm{E} 111^{\circ} 33^{\prime} 47.0^{\prime \prime}\end{array}$ & Secondary jungle & & $\sqrt{ }$ & & $\sqrt{ }$ & & $\sqrt{ }$ & $\sqrt{ }$ & & & & \\
\hline & 23 & $\begin{array}{l}\text { N01'29'34.1" } \\
\text { E111 } 32 ' 21.7^{\prime \prime}\end{array}$ & Secondary jungle, Acacia sp. & & & & $\sqrt{ }$ & & $\sqrt{ }$ & $\sqrt{ }$ & & & & \\
\hline & 24 & $\begin{array}{l}\text { N01³1'40.4" } \\
\text { E111 } 31^{\prime} 14.2^{\prime \prime}\end{array}$ & Secondary jungle & & & $\sqrt{ }$ & $\sqrt{ }$ & & $\sqrt{ }$ & $\sqrt{ }$ & & & & \\
\hline & 25 & $\begin{array}{l}\mathrm{N}^{\circ} 1^{\circ} 32^{\prime} 52.5^{\prime \prime} \\
\mathrm{E}_{111^{\circ} 29^{\prime} 13.5^{\prime \prime}}\end{array}$ & Secondary jungle & & & $\sqrt{ }$ & & & & $\sqrt{ }$ & & & & \\
\hline & 26 & $\begin{array}{l}\text { N01 } 35^{\circ} 01.2^{\prime \prime} \\
\text { E111 } 27^{\circ} 43.8^{\prime \prime}\end{array}$ & Secondary jungle & & & $\sqrt{ }$ & $\sqrt{ }$ & & & $\sqrt{ }$ & & & & \\
\hline & 27 & $\begin{array}{l}\text { N0136'28.1" } \\
\text { E111 } 21^{\circ} 10.1^{\prime \prime}\end{array}$ & Secondary jungle & & & & $\sqrt{ }$ & $\sqrt{ }$ & $\sqrt{ }$ & $\sqrt{ }$ & & & $\sqrt{ }$ & \\
\hline & 28 & 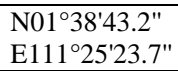 & Secondary jungle & & & & & & $\sqrt{ }$ & $\sqrt{ }$ & & & & \\
\hline & 29 & $\begin{array}{l}\mathrm{N} 01^{\circ} 41^{\prime} 08.5^{\prime \prime} \\
\mathrm{E} 111^{\circ} 25^{\prime} 51.9^{\prime \prime}\end{array}$ & Secondary jungle & & $\sqrt{ }$ & $\sqrt{ }$ & $\sqrt{ }$ & & $\sqrt{ }$ & $\sqrt{ }$ & & $\sqrt{ }$ & & \\
\hline & 30 & $\begin{array}{l}\text { N01 }{ }^{\circ} 43^{\prime} 24.0^{\prime \prime} \\
\text { E111 } 25 ' 22.4^{\prime \prime}\end{array}$ & Secondary jungle & & $\sqrt{ }$ & & $\sqrt{ }$ & & & $\sqrt{ }$ & & & & \\
\hline & 31 & $\begin{array}{l}\mathrm{N}^{\circ} 1^{\circ} 13^{\prime} 40.4^{\prime \prime} \\
\mathrm{E} 111^{\circ} 40^{\prime} 30.0^{\prime \prime}\end{array}$ & Mangiferae sp. & & & & & $\sqrt{ }$ & & $\sqrt{ }$ & & & & \\
\hline & 32 & $\begin{array}{l}\text { N01 }{ }^{\circ} 13^{\prime} 43.7^{\prime \prime} \\
\text { E111 } 42^{\prime} 33.9^{\prime \prime}\end{array}$ & Secondary jungle & & $\sqrt{ }$ & $\sqrt{ }$ & $\sqrt{ }$ & $\sqrt{ }$ & $\sqrt{ }$ & $\sqrt{ }$ & & & & \\
\hline & 33 & $\begin{array}{l}\text { N01 }{ }^{\circ} 12^{\prime} 05.6^{\prime \prime} \\
\text { E11 } 11^{\circ} 44^{\prime} 14.9^{\prime \prime}\end{array}$ & Secondary jungle & & & $\sqrt{ }$ & $\sqrt{ }$ & & $\sqrt{ }$ & $\sqrt{ }$ & & & & \\
\hline & 34 & $\begin{array}{l}\mathrm{N}^{\circ} 1^{\circ} 09^{\prime} 53.8^{\prime \prime} \\
\mathrm{E}^{\prime 1} 1^{\circ} 45^{\prime} 58.7^{\prime \prime}\end{array}$ & Mangiferae sp. & & & $\sqrt{ }$ & $\sqrt{ }$ & $\sqrt{ }$ & & $\sqrt{ }$ & & & & \\
\hline & 35 & $\begin{array}{l}\text { N01 }{ }^{\circ} 07^{\prime} 43.2^{\prime \prime} \\
\text { E111 } 47^{\prime} 34.5^{\prime \prime}\end{array}$ & Secondary jungle & & $\sqrt{ }$ & $\sqrt{ }$ & $\sqrt{ }$ & $\sqrt{ }$ & $\sqrt{ }$ & $\sqrt{ }$ & & & $\sqrt{ }$ & \\
\hline & 36 & $\begin{array}{l}\text { N01 } 05^{\circ} 27.5^{\prime \prime} \\
\text { E111 } 49 \text { '06.7" }\end{array}$ & Secondary jungle & & $\sqrt{ }$ & $\sqrt{ }$ & $\sqrt{ }$ & $\sqrt{ }$ & $\sqrt{ }$ & $\sqrt{ }$ & & & & \\
\hline & 37 & 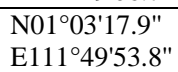 & Musa sp., Citrus sp. & & & $\sqrt{ }$ & $\sqrt{ }$ & $\sqrt{ }$ & $\sqrt{ }$ & $\sqrt{ }$ & & $\sqrt{ }$ & $\sqrt{ }$ & \\
\hline & 38 & $\begin{array}{l}\text { N01 }{ }^{\circ} 45^{\prime} 16.0^{\prime \prime} \\
\text { E111 } 23 \text { '36.6" }\end{array}$ & Secondary jungle & & & & $\sqrt{ }$ & & & $\sqrt{ }$ & & & & \\
\hline & 39 & $\begin{array}{l}\text { N01 }{ }^{\circ} 46^{\prime} 55.6^{\prime \prime} \\
\text { E111 } 21 \text { '38.1" }\end{array}$ & Secondary jungle & $\sqrt{ }$ & & $\sqrt{ }$ & $\sqrt{ }$ & $\sqrt{ }$ & $\sqrt{ }$ & $\sqrt{ }$ & $\sqrt{ }$ & & $\sqrt{ }$ & \\
\hline & 40 & $\begin{array}{l}\text { N01 }{ }^{\circ} 48^{\prime} 31.2^{\prime \prime} \\
\text { E111 } 19^{\prime} 39.8^{\prime \prime}\end{array}$ & Secondary jungle & & $\sqrt{ }$ & $\sqrt{ }$ & $\sqrt{ }$ & & & $\sqrt{ }$ & & $\sqrt{ }$ & $\sqrt{ }$ & \\
\hline & 41 & $\begin{array}{l}\text { N01 }{ }^{\circ} 51^{\prime} 06.7^{\prime \prime} \\
\text { E111 } 19^{\prime} 20.8^{\prime \prime}\end{array}$ & Secondary jungle & & $\sqrt{ }$ & & $\sqrt{ }$ & & & $\sqrt{ }$ & & $\sqrt{ }$ & & \\
\hline & 42 & $\begin{array}{l}\text { N01 }{ }^{\circ} 53^{\prime} 14.3^{\prime \prime} \\
\text { E111 } 21^{\prime} 03.2^{\prime \prime}\end{array}$ & Secondary jungle & & $\sqrt{ }$ & $\sqrt{ }$ & $\sqrt{ }$ & & $\sqrt{ }$ & $\sqrt{ }$ & & & & \\
\hline \multirow[t]{4}{*}{4} & 1 & $\begin{array}{l}\text { N01 }{ }^{\circ} 54^{\prime} 46.2^{\prime \prime} \\
\text { E111 } 22 ' 55.9^{\prime \prime}\end{array}$ & Secondary jungle & & & $\sqrt{ }$ & & & $\sqrt{ }$ & $\sqrt{ }$ & & & & \\
\hline & 2 & $\begin{array}{l}\text { N01 }{ }^{\circ} 55^{\prime} 38.9^{\prime \prime} \\
\text { E111 } 24^{\circ} 45.1^{\prime \prime}\end{array}$ & Secondary jungle & & & $\sqrt{ }$ & $\sqrt{ }$ & $\sqrt{ }$ & $\sqrt{ }$ & $\sqrt{ }$ & & & & \\
\hline & 3 & $\begin{array}{l}\text { N01 }{ }^{\circ} 57^{\prime} 39.7^{\prime \prime} \\
\text { E111 } 25^{\circ} 41.5^{\prime \prime}\end{array}$ & Secondary jungle & & & $\sqrt{ }$ & & & $\sqrt{ }$ & $\sqrt{ }$ & & $\sqrt{ }$ & & \\
\hline & 4 & $\begin{array}{l}\text { N01 }{ }^{\circ} 59^{\prime} 17.9^{\prime \prime} \\
\text { E111 } 26^{\prime} 34.2^{\prime \prime}\end{array}$ & Secondary jungle & $\sqrt{ }$ & $\sqrt{ }$ & & & $\sqrt{ }$ & & $\sqrt{ }$ & $\sqrt{ }$ & $\sqrt{ }$ & & \\
\hline
\end{tabular}




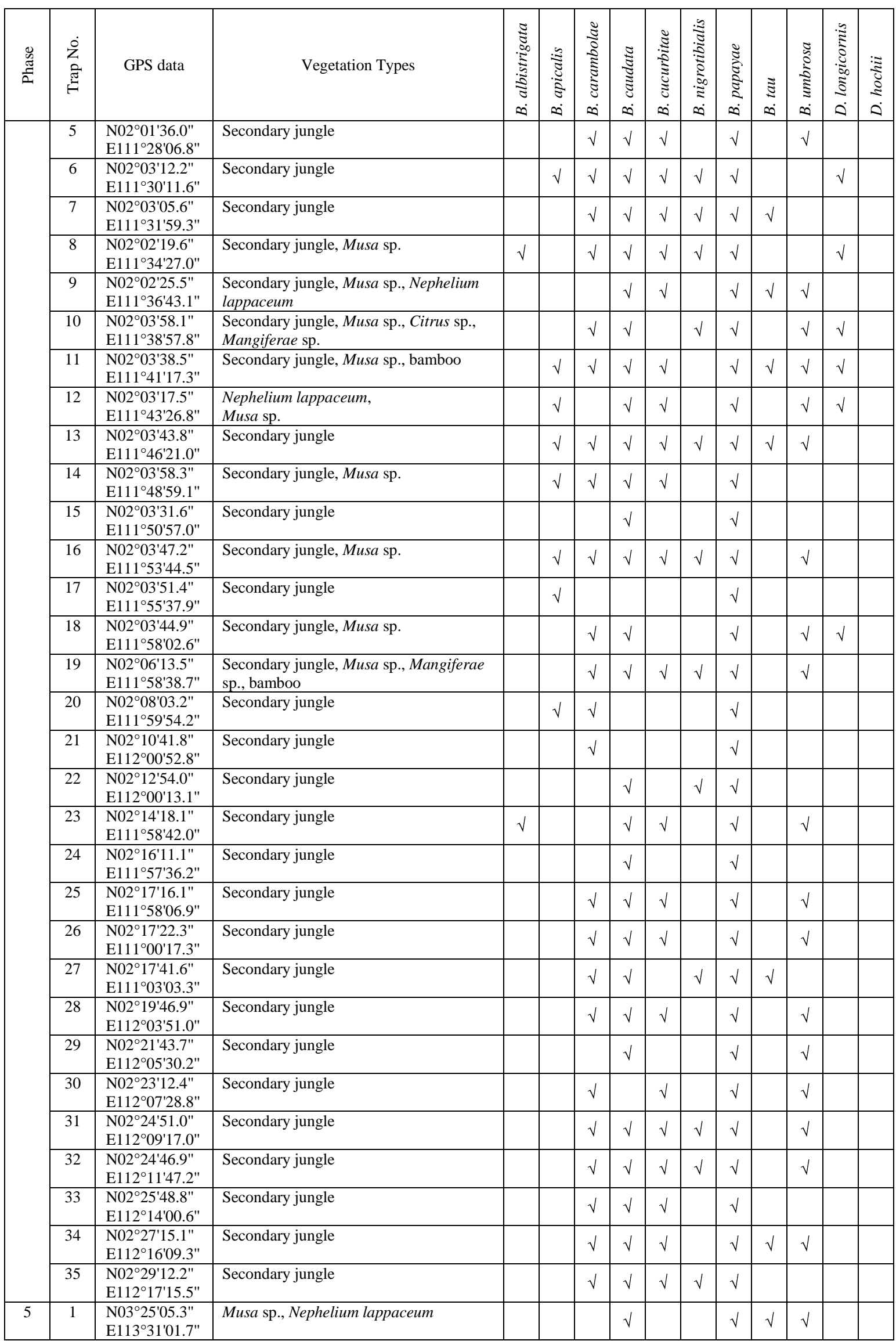




\begin{tabular}{|c|c|c|c|c|c|c|c|c|c|c|c|c|c|c|}
\hline 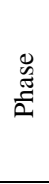 & 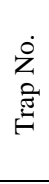 & GPS data & Vegetation Types & 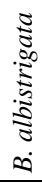 & 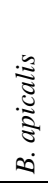 & 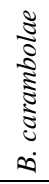 & $\begin{array}{c}\mathbb{Z} \\
\mathbb{Z} \\
\mathbb{8} \\
0 \\
0\end{array}$ & 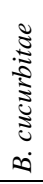 & 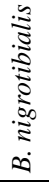 & 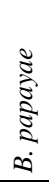 & 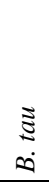 & $\begin{array}{r}\tilde{5} \\
\vdots \\
\vdots \\
\vdots \\
0 \\
\infty\end{array}$ & 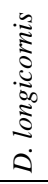 & $\begin{array}{l}: \vdots \\
\vdots \\
\Xi \\
0 \\
0\end{array}$ \\
\hline & 2 & 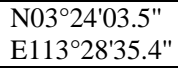 & Secondary jungle & & & & $\sqrt{ }$ & & & $\sqrt{ }$ & & $\sqrt{ }$ & & \\
\hline & 3 & 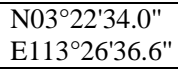 & $\begin{array}{l}\text { Secondary jungle, Musa sp., Citrus sp., } \\
\text { Mangiferae sp. }\end{array}$ & & & & $\sqrt{ }$ & $\sqrt{ }$ & & $\sqrt{ }$ & & $\sqrt{ }$ & & \\
\hline & 4 & 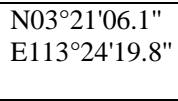 & $\begin{array}{l}\text { Musa sp., Cocos nucifera, Lansium } \\
\text { domesticum, Syzygium samarangense, } \\
\text { Elaies guineensis }\end{array}$ & & & & $\sqrt{ }$ & $\sqrt{ }$ & & $\sqrt{ }$ & & $\sqrt{ }$ & & \\
\hline & 5 & 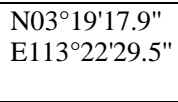 & $\begin{array}{l}\text { Canarium odontophyllum, terap, } \\
\text { Nephelium lappaceum, Syzygium } \\
\text { samarangense }\end{array}$ & & & & $\sqrt{ }$ & $\sqrt{ }$ & $\sqrt{ }$ & $\sqrt{ }$ & & $\sqrt{ }$ & & \\
\hline & 6 & $\begin{array}{l}\text { N03 } 17^{\prime} 19.1^{\prime \prime} \\
\text { E113'20'39.9" }\end{array}$ & Secondary jungle, Elaies guineensis & & $\sqrt{ }$ & & $\sqrt{ }$ & $\sqrt{ }$ & & $\sqrt{ }$ & & & & \\
\hline & 7 & 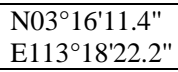 & $\begin{array}{l}\text { Secondary jungle, Mangiferae sp., } \\
\text { Nephelium lappaceum, terap }\end{array}$ & & & & $\sqrt{ }$ & $\sqrt{ }$ & & $\sqrt{ }$ & $\sqrt{ }$ & $\sqrt{ }$ & & \\
\hline & 8 & $\begin{array}{l}\mathrm{N}^{\circ} 3^{\circ} 14^{\prime} 48.2^{\prime \prime} \\
\mathrm{E} 113^{\circ} 16^{\prime} 03.8^{\prime \prime}\end{array}$ & $\begin{array}{l}\text { Secondary jungle, Musa sp., Artocarpus } \\
\text { integer }\end{array}$ & & & & $\sqrt{ }$ & $\sqrt{ }$ & & $\sqrt{ }$ & & $\sqrt{ }$ & & \\
\hline & 9 & $\begin{array}{l}\text { N03 } 14^{\prime} 27.6^{\prime \prime} \\
\text { E113 } 13^{\prime} 44.7^{\prime \prime}\end{array}$ & Secondary jungle & & & $\sqrt{ }$ & $\sqrt{ }$ & $\sqrt{ }$ & & $\sqrt{ }$ & & $\sqrt{ }$ & & \\
\hline & 10 & 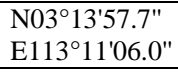 & $\begin{array}{l}\text { Secondary jungle, Canarium } \\
\text { odontophyllum, Cocos nucifera }\end{array}$ & & & & $\sqrt{ }$ & $\sqrt{ }$ & & $\sqrt{ }$ & $\sqrt{ }$ & $\sqrt{ }$ & & \\
\hline & 11 & $\begin{array}{l}\mathrm{N}^{\circ} 3^{\circ} 13^{\prime} 03.7^{\prime \prime} \\
\mathrm{E} 113^{\circ} 08^{\prime} 48.1^{\prime \prime}\end{array}$ & Mangiferae sp., Artocarpus integer, terap & $\sqrt{ }$ & & & $\sqrt{ }$ & $\sqrt{ }$ & & $\sqrt{ }$ & & $\sqrt{ }$ & & \\
\hline & 12 & 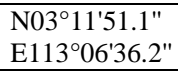 & Secondary jungle & $\sqrt{ }$ & & & $\sqrt{ }$ & $\sqrt{ }$ & & $\sqrt{ }$ & & $\sqrt{ }$ & & \\
\hline & 13 & 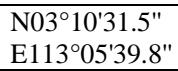 & Secondary jungle & & & & $\sqrt{ }$ & & & $\sqrt{ }$ & & $\sqrt{ }$ & & \\
\hline & 14 & 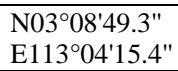 & Secondary jungle & $\sqrt{ }$ & & & $\sqrt{ }$ & & & $\sqrt{ }$ & & $\sqrt{ }$ & & \\
\hline & 15 & $\begin{array}{l}\mathrm{N}^{\circ} 03^{\circ} 26.3^{\prime \prime} \\
\mathrm{E} 113^{\circ} 02^{\prime} 01.6^{\prime \prime}\end{array}$ & Secondary jungle & & & & $\sqrt{ }$ & $\sqrt{ }$ & & $\sqrt{ }$ & & $\sqrt{ }$ & & \\
\hline & 16 & $\begin{array}{l}\mathrm{N}^{\circ} 03^{\circ} 38.5^{\prime \prime} \\
\mathrm{E} 113^{\circ} 00^{\prime} 18.8^{\prime \prime}\end{array}$ & Secondary jungle & & & $\sqrt{ }$ & $\sqrt{ }$ & $\sqrt{ }$ & $\sqrt{ }$ & $\sqrt{ }$ & & & & \\
\hline & 17 & $\begin{array}{l}\text { N03 } 04^{\circ} 40.7^{\prime \prime} \\
\text { E112 } 58^{\prime} 12.5^{\prime \prime}\end{array}$ & Secondary jungle & & & & $\sqrt{ }$ & $\sqrt{ }$ & & $\sqrt{ }$ & & $\sqrt{ }$ & & \\
\hline & 18 & $\begin{array}{l}\text { N03 } 03 \text { 'o3.3" } \\
\text { E112 } 56^{\prime} 16.4^{\prime \prime}\end{array}$ & Secondary jungle & & & $\sqrt{ }$ & $\sqrt{ }$ & & & $\sqrt{ }$ & & $\sqrt{ }$ & & \\
\hline & 19 & $\begin{array}{l}\text { N03ㅇํ'55.8" } \\
\text { E11255'12.1" }\end{array}$ & Secondary jungle & & & & $\sqrt{ }$ & $\sqrt{ }$ & & $\sqrt{ }$ & & $\sqrt{ }$ & & \\
\hline & 20 & $\begin{array}{l}\text { N0258'33.1" } \\
\text { E112॰53'57.3" }\end{array}$ & Secondary jungle, Zea mays & & & $\sqrt{ }$ & $\sqrt{ }$ & $\sqrt{ }$ & $\sqrt{ }$ & $\sqrt{ }$ & & $\sqrt{ }$ & & \\
\hline & 21 & $\begin{array}{l}\text { N02 }{ }^{\circ} 56^{\prime} 16.5^{\prime \prime} \\
\text { E112 } 53^{\prime} 10.6^{\prime \prime}\end{array}$ & Secondary jungle & & & $\sqrt{ }$ & $\sqrt{ }$ & $\sqrt{ }$ & & $\sqrt{ }$ & & $\sqrt{ }$ & & \\
\hline & 22 & $\begin{array}{l}\text { N02 } 54 ' 26.4^{\prime \prime} \\
\text { E112 } 51^{\prime} 25.0^{\prime \prime}\end{array}$ & Secondary jungle & & & & $\sqrt{ }$ & $\sqrt{ }$ & & $\sqrt{ }$ & & $\sqrt{ }$ & & \\
\hline & 23 & 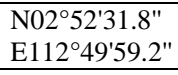 & Secondary jungle & & & & & & & $\sqrt{ }$ & & $\sqrt{ }$ & & \\
\hline & 24 & $\begin{array}{l}\text { N02 } 511^{\prime} 27.8^{\prime \prime} \\
\text { E112 } 47^{\prime} 48.8^{\prime \prime}\end{array}$ & Secondary jungle, Elaies guineensis & & & & $\sqrt{ }$ & $\sqrt{ }$ & & $\sqrt{ }$ & $\sqrt{ }$ & & & \\
\hline & 25 & $\begin{array}{l}\text { N02 } 50^{\circ} 03.8^{\prime \prime} \\
\text { E11245'44.1" }\end{array}$ & Secondary jungle & & & $\sqrt{ }$ & $\sqrt{ }$ & $\sqrt{ }$ & & $\sqrt{ }$ & & $\sqrt{ }$ & & \\
\hline & 26 & $\begin{array}{l}\text { N02 } 48^{\prime} 02.3^{\prime \prime} \\
\mathrm{E} 112^{\circ} 44^{\prime} 19.1^{\prime \prime}\end{array}$ & $\begin{array}{l}\text { Secondary jungle, Musa sp., Saurapus } \\
\text { androgynous }\end{array}$ & & & $\sqrt{ }$ & $\sqrt{ }$ & $\sqrt{ }$ & $\sqrt{ }$ & $\sqrt{ }$ & & $\sqrt{ }$ & & \\
\hline & 27 & $\begin{array}{l}\text { N02 } 466^{\prime} 17.3^{\prime \prime} \\
\text { E112²41'59.7" }\end{array}$ & Secondary jungle & $\sqrt{ }$ & & $\sqrt{ }$ & $\sqrt{ }$ & $\sqrt{ }$ & $\sqrt{ }$ & $\sqrt{ }$ & & $\sqrt{ }$ & & \\
\hline & 28 & $\begin{array}{l}\text { N02 } 44 ' 53.9^{\prime \prime} \\
\text { E112³9'52.3" }\end{array}$ & Secondary jungle & & & $\sqrt{ }$ & $\sqrt{ }$ & $\sqrt{ }$ & $\sqrt{ }$ & $\sqrt{ }$ & & $\sqrt{ }$ & $\sqrt{ }$ & \\
\hline & 29 & $\begin{array}{l}\mathrm{N} 02^{\circ} 42^{\prime} 35.2^{\prime \prime} \\
\mathrm{E} 112^{\circ} 40^{\prime} 32.8^{\prime \prime}\end{array}$ & $\begin{array}{l}\text { Secondary jungle, Musa sp., Elaies } \\
\text { guineensis, Capsicum sp. }\end{array}$ & & & $\sqrt{ }$ & $\sqrt{ }$ & $\sqrt{ }$ & $\sqrt{ }$ & $\sqrt{ }$ & & $\sqrt{ }$ & & \\
\hline & 30 & 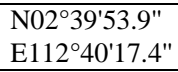 & $\begin{array}{l}\text { Secondary jungle, Musa sp., Mangiferae } \\
\text { sp. }\end{array}$ & & & $\sqrt{ }$ & $\sqrt{ }$ & & & $\sqrt{ }$ & & & & \\
\hline & 31 & $\begin{array}{l}\text { N02³7'37.8" } \\
\text { E112³8'49.5" }\end{array}$ & Elaies guineensis & & & & $\sqrt{ }$ & & & $\sqrt{ }$ & & & & \\
\hline & 32 & $\begin{array}{l}\mathrm{N} 02^{\circ} 36^{\prime} 28.3^{\prime \prime} \\
\mathrm{E} 112^{\circ} 36^{\prime} 41.8^{\prime \prime}\end{array}$ & $\begin{array}{l}\text { Acacia sp., Musa sp., Mangiferae sp., } \\
\text { Carica papaya }\end{array}$ & & & & & & & $\sqrt{ }$ & & $\sqrt{ }$ & & \\
\hline & 33 & $\begin{array}{l}\text { N02³6'05.6" } \\
\text { E112 } 34^{\prime} 07.5^{\prime \prime}\end{array}$ & Secondary jungle, Musa sp. & & & $\sqrt{ }$ & & & $\sqrt{ }$ & $\sqrt{ }$ & & $\sqrt{ }$ & & \\
\hline
\end{tabular}




\begin{tabular}{|c|c|c|c|c|c|c|c|c|c|c|c|c|c|c|}
\hline 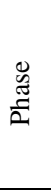 & 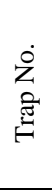 & GPS data & Vegetation Types & 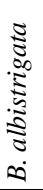 & 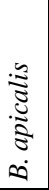 & 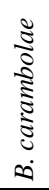 & 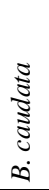 & 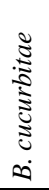 & 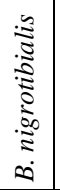 & 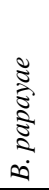 & $\begin{array}{c}3 \\
\infty \\
0\end{array}$ & $\begin{array}{l}\square \\
\vdots \\
\vdots \\
\vdots \\
\vdots \\
\infty \\
\infty\end{array}$ & 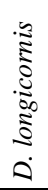 & $\begin{array}{l}: \vdots \\
\vdots \\
0 \\
\vdots \\
0\end{array}$ \\
\hline & 34 & $\begin{array}{l}\text { N02 } 34^{\prime} 01.7^{\prime \prime} \\
\text { E112 } 32 ' 33.0^{\prime \prime}\end{array}$ & Secondary jungle & & & $\sqrt{ }$ & $\sqrt{ }$ & & $\sqrt{ }$ & $\sqrt{ }$ & & & & \\
\hline & 35 & $\begin{array}{l}\text { N02 } 33 \prime 23.9^{\prime \prime} \\
\text { E112 } 30 ' 31.4^{\prime \prime}\end{array}$ & Secondary jungle & & & $\sqrt{ }$ & $\sqrt{ }$ & $\sqrt{ }$ & $\sqrt{ }$ & $\sqrt{ }$ & & $\sqrt{ }$ & & \\
\hline & 36 & $\begin{array}{l}\text { N02 } 33 ' 37.5^{\prime \prime} \\
\mathrm{E} 112^{\circ} 27^{\prime} 59.6^{\prime \prime}\end{array}$ & Secondary jungle & & & $\sqrt{ }$ & & & & $\sqrt{ }$ & $\sqrt{ }$ & & & \\
\hline & 37 & $\begin{array}{l}\mathrm{N} 02^{\circ} 33^{\prime} 52.7^{\prime \prime} \\
\mathrm{E} 112^{\circ} 25^{\prime} 22.4^{\prime \prime}\end{array}$ & Secondary jungle & & & $\sqrt{ }$ & $\sqrt{ }$ & $\sqrt{ }$ & & $\sqrt{ }$ & & & & \\
\hline & 38 & $\begin{array}{l}\mathrm{N} 02^{\circ} 33^{\prime} 07.2^{\prime \prime} \\
\mathrm{E} 112^{\circ} 23^{\prime} 00.1^{\prime \prime}\end{array}$ & $\begin{array}{l}\text { Secondary jungle, Mangiferae sp., } \\
\text { Nephelium lappaceum, Syzygium } \\
\text { samarangense }\end{array}$ & & & $\sqrt{ }$ & $\sqrt{ }$ & & & $\sqrt{ }$ & & & & \\
\hline & 39 & $\begin{array}{l}\mathrm{N} 02^{\circ} 32^{\prime} 26.6^{\prime \prime} \\
\mathrm{E} 112^{\circ} 20^{\prime} 29.1^{\prime \prime}\end{array}$ & Secondary jungle & & & & $\sqrt{ }$ & & & $\sqrt{ }$ & & & & \\
\hline & 40 & $\begin{array}{l}\text { N02 }{ }^{\circ} 30^{\prime} 51.1^{\prime \prime} \\
\text { E112 } 12^{\circ} 50.0^{\prime \prime}\end{array}$ & Secondary jungle, Artocarpus integer & & & $\sqrt{ }$ & $\sqrt{ }$ & $\sqrt{ }$ & & $\sqrt{ }$ & & & & \\
\hline \multirow[t]{24}{*}{6} & 1 & $\begin{array}{l}\text { N043' } 55.5^{\prime \prime} \\
\text { E114 } 04^{\prime} 12.5^{\prime \prime}\end{array}$ & Secondary jungle & $\sqrt{ }$ & & & $\sqrt{ }$ & & & $\sqrt{ }$ & & & & \\
\hline & 2 & $\begin{array}{l}\mathrm{N} 04^{\circ} 32^{\prime} 52.2^{\prime \prime} \\
\mathrm{E} 114^{\circ} 03^{\prime} 28.9^{\prime \prime}\end{array}$ & Secondary jungle & & $\sqrt{ }$ & & $\sqrt{ }$ & $\sqrt{ }$ & & $\sqrt{ }$ & & & & \\
\hline & 3 & 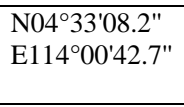 & $\begin{array}{l}\text { Musa sp., Cocos nucifera, Artocarpus } \\
\text { heterophyllus, Mangiferae sp., Saurapus } \\
\text { androgynous }\end{array}$ & $\sqrt{ }$ & $\sqrt{ }$ & & $\sqrt{ }$ & $\sqrt{ }$ & & $\sqrt{ }$ & & $\sqrt{ }$ & & \\
\hline & 4 & $\begin{array}{l}\text { N0431'27.8" } \\
\text { E113 } 59^{\prime} 15.3^{\prime \prime}\end{array}$ & Secondary jungle & $\sqrt{ }$ & & & $\sqrt{ }$ & $\sqrt{ }$ & & $\sqrt{ }$ & & & & \\
\hline & 5 & 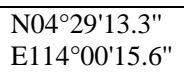 & Musa sp., Artocarpus heterophyllus & $\sqrt{ }$ & & & $\sqrt{ }$ & & & $\sqrt{ }$ & & $\sqrt{ }$ & & \\
\hline & 6 & $\begin{array}{l}\text { N04 } 26^{\prime} 33.9^{\prime \prime} \\
\text { E114 } 00^{\prime} 37.5^{\prime \prime}\end{array}$ & Musa sp., Mangiferae sp. & $\sqrt{ }$ & & & $\sqrt{ }$ & $\sqrt{ }$ & & $\sqrt{ }$ & & $\sqrt{ }$ & & \\
\hline & 7 & 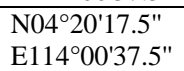 & $\begin{array}{l}\text { Citrus sp., Citrus maxima, Syzygium } \\
\text { samarangense, leafy vegetable }\end{array}$ & $\sqrt{ }$ & $\sqrt{ }$ & & $\sqrt{ }$ & $\sqrt{ }$ & & $\sqrt{ }$ & & $\sqrt{ }$ & & \\
\hline & 8 & 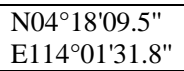 & Secondary jungle & $\sqrt{ }$ & & & $\sqrt{ }$ & $\sqrt{ }$ & & $\sqrt{ }$ & & $\sqrt{ }$ & & \\
\hline & 9 & 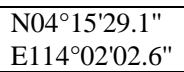 & Secondary jungle, Musa sp. & $\sqrt{ }$ & & $\sqrt{ }$ & & & & $\sqrt{ }$ & & $\sqrt{ }$ & & \\
\hline & 10 & 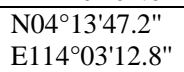 & Secondary jungle & & & & & & & $\sqrt{ }$ & & $\sqrt{ }$ & & \\
\hline & 11 & $\begin{array}{l}\text { N041'ㅇ․ } \\
\text { E11 } 14^{\circ} 02^{\prime \prime} 20.9^{\prime \prime}\end{array}$ & Secondary jungle & & & & $\sqrt{ }$ & & & $\sqrt{ }$ & & $\sqrt{ }$ & & \\
\hline & 12 & 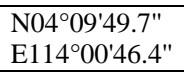 & Secondary jungle & $\sqrt{ }$ & & & & $\sqrt{ }$ & & $\sqrt{ }$ & & & & \\
\hline & 13 & 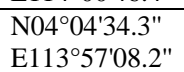 & Secondary jungle, Averrhoa carambola & $\sqrt{ }$ & $\sqrt{ }$ & $\sqrt{ }$ & $\sqrt{ }$ & & $\sqrt{ }$ & $\sqrt{ }$ & & $\sqrt{ }$ & $\sqrt{ }$ & \\
\hline & 14 & $\begin{array}{l}\mathrm{N}^{\circ} 04^{\circ} 02^{\prime} 03.0^{\prime \prime} \\
\mathrm{E} 113^{\circ} 56^{\prime} 24.8^{\prime \prime}\end{array}$ & Citrus sp., terap & $\sqrt{ }$ & $\sqrt{ }$ & $\sqrt{ }$ & $\sqrt{ }$ & $\sqrt{ }$ & & $\sqrt{ }$ & & $\sqrt{ }$ & & \\
\hline & 15 & $\begin{array}{l}\mathrm{N}^{\circ} 53^{\circ} 59^{\prime} 32.0^{\prime \prime} \\
\mathrm{E} 113^{\circ} 56^{\prime} 27.9^{\prime \prime}\end{array}$ & $\begin{array}{l}\text { Cocos nucifera, Artocarpus } \\
\text { heterophyllus, Nephelium lappaceum }\end{array}$ & & & $\sqrt{ }$ & $\sqrt{ }$ & $\sqrt{ }$ & & $\sqrt{ }$ & $\sqrt{ }$ & $\sqrt{ }$ & $\sqrt{ }$ & \\
\hline & 16 & 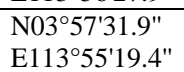 & $\begin{array}{l}\text { Durio sp., Artocarpus heterophyllus, } \\
\text { terap }\end{array}$ & & & $\sqrt{ }$ & $\sqrt{ }$ & & $\sqrt{ }$ & $\sqrt{ }$ & & & & \\
\hline & 17 & $\begin{array}{l}\text { N03 } 54^{\prime} 13.8^{\prime \prime} \\
\text { E113 } 54^{\prime} 04.9^{\prime \prime}\end{array}$ & Secondary jungle & $\sqrt{ }$ & & $\sqrt{ }$ & $\sqrt{ }$ & & & $\sqrt{ }$ & & & $\sqrt{ }$ & \\
\hline & 18 & $\begin{array}{l}\mathrm{N}^{\circ} 46^{\circ} 59.3^{\prime \prime} \\
\text { E113 } 49^{\circ} 49.4^{\prime \prime}\end{array}$ & $\begin{array}{l}\text { Musa sp., Psidium sp., Nephelium } \\
\text { lappaceum, Mangiferae pajang }\end{array}$ & & & $\sqrt{ }$ & $\sqrt{ }$ & $\sqrt{ }$ & $\sqrt{ }$ & $\sqrt{ }$ & $\sqrt{ }$ & $\sqrt{ }$ & & \\
\hline & 19 & $\begin{array}{l}\mathrm{N}^{\circ} 3^{\circ} 44^{\prime} 55.0^{\prime \prime} \\
\text { E113 } 45^{\prime} 54.8^{\prime \prime}\end{array}$ & Durio sp., Nephelium lappaceum, terap & & & & $\sqrt{ }$ & $\sqrt{ }$ & & $\sqrt{ }$ & $\sqrt{ }$ & $\sqrt{ }$ & & \\
\hline & 20 & $\begin{array}{l}\text { N034' } 38.6^{\prime \prime} \\
\text { E113 } 45^{\prime} 54.8^{\prime \prime}\end{array}$ & Musa sp., Artocarpus integer & & & $\sqrt{ }$ & $\sqrt{ }$ & & & $\sqrt{ }$ & & $\sqrt{ }$ & $\sqrt{ }$ & \\
\hline & 21 & $\begin{array}{l}\text { N034ㄴ' } 02.9^{\prime \prime} \\
\text { E1134ㄴ' } 26.0^{\prime \prime}\end{array}$ & Secondary jungle & & & $\sqrt{ }$ & $\sqrt{ }$ & $\sqrt{ }$ & & $\sqrt{ }$ & $\sqrt{ }$ & & & \\
\hline & 22 & $\begin{array}{l}\mathrm{N}^{\circ} 33^{\circ} 48.9^{\prime \prime} \\
\text { E113 } 42^{\prime} 58.5^{\prime \prime}\end{array}$ & $\begin{array}{l}\text { Cocos nucifera, Musa sp., Mangiferae } \\
\text { sp., Psidium sp. }\end{array}$ & & & $\sqrt{ }$ & $\sqrt{ }$ & & & $\sqrt{ }$ & & $\sqrt{ }$ & & \\
\hline & 23 & $\begin{array}{l}\mathrm{N}^{\circ} 33^{\circ} 36^{\prime} 52.7^{\prime \prime} \\
\text { E113 } 40^{\prime} 59.9^{\prime \prime}\end{array}$ & Secondary jungle, Musa sp. & & & $\sqrt{ }$ & $\sqrt{ }$ & & & $\sqrt{ }$ & & & & \\
\hline & 24 & $\begin{array}{l}\mathrm{N}^{\circ} 33^{\circ} 35^{\prime} 05.7^{\prime \prime} \\
\mathrm{E} 113^{\circ} 39^{\prime} 02.4^{\prime \prime}\end{array}$ & $\begin{array}{l}\text { Musa sp., Psidium sp., Nephelium } \\
\text { lappaceum, Mangiferae pajang }\end{array}$ & & & $\sqrt{ }$ & $\sqrt{ }$ & & $\sqrt{ }$ & $\sqrt{ }$ & & & & \\
\hline
\end{tabular}




\begin{tabular}{|c|c|c|c|c|c|c|c|c|c|c|c|c|c|c|}
\hline$\frac{\mathscr{D}}{\tilde{E}}$ & 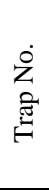 & GPS data & Vegetation Types & 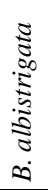 & 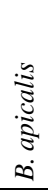 & 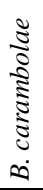 & 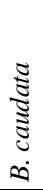 & 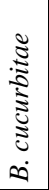 & 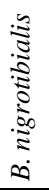 & 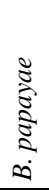 & $\underset{\substack{3 \\
\infty}}{\infty}$ & 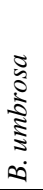 & 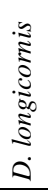 & 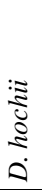 \\
\hline & 25 & $\begin{array}{l}{\mathrm{N} 03^{\circ} 32^{\prime} 53.8^{\prime \prime}}^{\mathrm{E} 113^{\circ} 37^{\prime} 23.4^{\prime \prime}} \\
\end{array}$ & Durio sp., Nephelium lappaceum & & & $\sqrt{ }$ & $\sqrt{ }$ & & $\sqrt{ }$ & $\sqrt{ }$ & & & & \\
\hline & 26 & $\begin{array}{l}\text { N0330'59.7" } \\
\text { E11336'00.7" }\end{array}$ & Durio sp., mempalam & & & $\sqrt{ }$ & & & & $\sqrt{ }$ & & $\sqrt{ }$ & & \\
\hline & 27 & $\begin{array}{l}\text { N03 }^{\circ} 28^{\prime} 33.3^{\prime \prime} \\
\text { E113 } 34^{\prime} 10.7^{\prime \prime}\end{array}$ & Secondary jungle & & & $\sqrt{ }$ & $\sqrt{ }$ & & & $\sqrt{ }$ & & & & \\
\hline & 28 & 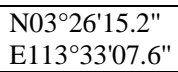 & Musa sp. & & & $\sqrt{ }$ & $\sqrt{ }$ & $\sqrt{ }$ & $\sqrt{ }$ & $\sqrt{ }$ & & & & \\
\hline \multirow[t]{23}{*}{7} & 1 & 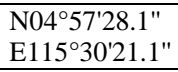 & Psidium sp., Nephelium lappaceum & $\sqrt{ }$ & & $\sqrt{ }$ & $\sqrt{ }$ & $\sqrt{ }$ & $\sqrt{ }$ & $\sqrt{ }$ & $\sqrt{ }$ & & & \\
\hline & 2 & 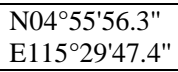 & Secondary jungle & $\sqrt{ }$ & & $\sqrt{ }$ & $\sqrt{ }$ & & $\sqrt{ }$ & $\sqrt{ }$ & & & & \\
\hline & 3 & $\begin{array}{l}\text { N04'54'46.8" } \\
\text { E11527'44.4" }\end{array}$ & Secondary jungle & $\sqrt{ }$ & & $\sqrt{ }$ & & & $\sqrt{ }$ & $\sqrt{ }$ & & & & \\
\hline & 4 & $\begin{array}{l}\text { N04 } 52^{\prime} 19.0^{\prime \prime} \\
\mathrm{E} 115^{\circ} 26^{\prime} 29.7^{\prime \prime}\end{array}$ & Secondary jungle & $\sqrt{ }$ & & $\sqrt{ }$ & $\sqrt{ }$ & & $\sqrt{ }$ & $\sqrt{ }$ & $\sqrt{ }$ & & & \\
\hline & 5 & $\begin{array}{l}\text { N0450'24.7" } \\
\text { E115'26'11.4" }\end{array}$ & Secondary jungle & & & $\sqrt{ }$ & $\sqrt{ }$ & $\sqrt{ }$ & $\sqrt{ }$ & $\sqrt{ }$ & & $\sqrt{ }$ & & \\
\hline & 6 & $\begin{array}{l}\text { N04 }{ }^{\circ} 48^{\prime} 36.6^{\prime \prime} \\
\text { E115 } 25^{\circ} 44.7^{\prime \prime}\end{array}$ & Musa sp., Hylocereus sp., mata kucing & $\sqrt{ }$ & & $\sqrt{ }$ & $\sqrt{ }$ & $\sqrt{ }$ & $\sqrt{ }$ & $\sqrt{ }$ & $\sqrt{ }$ & & & \\
\hline & 7 & 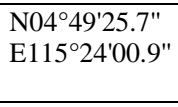 & $\begin{array}{l}\text { Theobroma cocoa, Dimocarpus longan, } \\
\text { australian laichi, Nephelium lappaceum, } \\
\text { lombok }\end{array}$ & $\sqrt{ }$ & & $\sqrt{ }$ & $\sqrt{ }$ & $\sqrt{ }$ & $\sqrt{ }$ & $\sqrt{ }$ & $\sqrt{ }$ & & & \\
\hline & 8 & 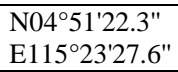 & Vitex pinnata & $\sqrt{ }$ & & $\sqrt{ }$ & $\sqrt{ }$ & $\sqrt{ }$ & $\sqrt{ }$ & $\sqrt{ }$ & $\sqrt{ }$ & & & \\
\hline & 9 & $\begin{array}{l}\text { N0450'52.9" } \\
\text { E115'21'10.7" }\end{array}$ & $\begin{array}{l}\text { Musa sp., Cocos nucifera, Mangiferae } \\
\text { sp., Nephelium lappaceum }\end{array}$ & $\sqrt{ }$ & & $\sqrt{ }$ & $\sqrt{ }$ & $\sqrt{ }$ & $\sqrt{ }$ & $\sqrt{ }$ & $\sqrt{ }$ & $\sqrt{ }$ & & \\
\hline & 10 & $\begin{array}{l}\text { N04ㅇ' } 38.4^{\prime \prime} \\
\text { E115॰18'53.5" }\end{array}$ & $\begin{array}{l}\text { Musa sp., Artocarpus heterophyllus, } \\
\text { Durio sp. belanda, Citrus maxima }\end{array}$ & & & $\sqrt{ }$ & $\sqrt{ }$ & & $\sqrt{ }$ & $\sqrt{ }$ & $\sqrt{ }$ & $\sqrt{ }$ & & \\
\hline & 11 & $\begin{array}{l}\text { N04 } 47^{\prime} 48.1^{\prime \prime} \\
\text { E115 } 16^{\prime} 50.9^{\prime \prime}\end{array}$ & $\begin{array}{l}\text { Musa sp., Citrus sp., Psidium sp., } \\
\text { Mangiferae sp. }\end{array}$ & & & $\sqrt{ }$ & $\sqrt{ }$ & $\sqrt{ }$ & $\sqrt{ }$ & $\sqrt{ }$ & $\sqrt{ }$ & $\sqrt{ }$ & & \\
\hline & 12 & $\begin{array}{l}\mathrm{N} 04^{\circ} 46^{\prime} 31.8^{\prime \prime} \\
\mathrm{E} 115^{\circ} 15^{\prime} 03.8^{\prime \prime}\end{array}$ & $\begin{array}{l}\text { Musa sp., Artocarpus heterophyllus, } \\
\text { Psidium sp., Artocarpus heterophyllus }\end{array}$ & & & $\sqrt{ }$ & $\sqrt{ }$ & $\sqrt{ }$ & & $\sqrt{ }$ & & & & \\
\hline & 13 & 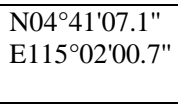 & $\begin{array}{l}\text { Musa sp., Cocos nucifera, Psidium sp., } \\
\text { Citrus } \text { sp. maxima, Nephelium } \\
\text { lappaceum }\end{array}$ & & & $\sqrt{ }$ & $\sqrt{ }$ & $\sqrt{ }$ & $\sqrt{ }$ & $\sqrt{ }$ & $\sqrt{ }$ & $\sqrt{ }$ & & \\
\hline & 14 & $\begin{array}{l}\text { N04 } 42^{\prime} 05.9^{\prime \prime} \\
\text { E115 } 00^{\prime} 20.0^{\prime \prime}\end{array}$ & $\begin{array}{l}\text { Musa sp., Psidium sp., Artocarpus } \\
\text { heterophyllus, Capsicum sp., Luffa sp., } \\
\text { leafy vegetables }\end{array}$ & $\sqrt{ }$ & & $\sqrt{ }$ & $\sqrt{ }$ & $\sqrt{ }$ & & $\sqrt{ }$ & $\sqrt{ }$ & $\sqrt{ }$ & & \\
\hline & 15 & $\begin{array}{l}\text { N044'36.8" } \\
\text { E11500'51.2" }\end{array}$ & $\begin{array}{l}\text { Musa sp., Artocarpus heterophyllus, } \\
\text { Syzygium samarangense, leafy } \\
\text { vegetables, Luffa sp., Capsicum sp. }\end{array}$ & $\sqrt{ }$ & & & $\sqrt{ }$ & $\sqrt{ }$ & $\sqrt{ }$ & $\sqrt{ }$ & & $\sqrt{ }$ & & \\
\hline & 16 & $\begin{array}{l}\mathrm{N} 04^{\circ} 43^{\prime} 20.3^{\prime \prime} \\
\mathrm{E} 114^{\circ} 59^{\prime} 17.8^{\prime \prime}\end{array}$ & Mangiferae sp., Averrhoa carambola & $\sqrt{ }$ & & & $\sqrt{ }$ & $\sqrt{ }$ & $\sqrt{ }$ & $\sqrt{ }$ & $\sqrt{ }$ & $\sqrt{ }$ & & \\
\hline & 17 & 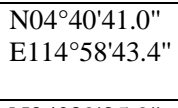 & $\begin{array}{l}\text { Musa sp., Citrus sp., Hylocereus sp., } \\
\text { Psidium sp., Mangiferae sp., Carica } \\
\text { papaya }\end{array}$ & $\sqrt{ }$ & & & $\sqrt{ }$ & $\sqrt{ }$ & $\sqrt{ }$ & $\sqrt{ }$ & & & & \\
\hline & 18 & $\begin{array}{l}\text { N0439'25.9" } \\
\text { E1145' } 15.1^{\prime \prime}\end{array}$ & Musa sp., Manilkara zapota, Psidium sp. & $\sqrt{ }$ & & $\sqrt{ }$ & $\sqrt{ }$ & $\sqrt{ }$ & $\sqrt{ }$ & $\sqrt{ }$ & & $\sqrt{ }$ & & \\
\hline & 19 & 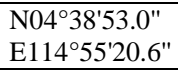 & Cocos nucifera, Psidium sp. & & & & $\sqrt{ }$ & & $\sqrt{ }$ & $\sqrt{ }$ & $\sqrt{ }$ & $\sqrt{ }$ & & \\
\hline & 20 & $\begin{array}{l}\text { N04³8'39.4" } \\
\text { E1145' } 22.7^{\prime \prime}\end{array}$ & Musa sp., Mangiferae sp. & $\sqrt{ }$ & $\sqrt{ }$ & $\sqrt{ }$ & $\sqrt{ }$ & $\sqrt{ }$ & $\sqrt{ }$ & $\sqrt{ }$ & $\sqrt{ }$ & $\sqrt{ }$ & & \\
\hline & 21 & 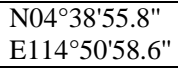 & $\begin{array}{l}\text { Musa sp., Citrus sp., Nephelium } \\
\text { lappaceum }\end{array}$ & $\sqrt{ }$ & $\sqrt{ }$ & & $\sqrt{ }$ & & $\sqrt{ }$ & $\sqrt{ }$ & $\sqrt{ }$ & $\sqrt{ }$ & & \\
\hline & 22 & $\begin{array}{l}\text { N044ㅇ'06.1" } \\
\text { E114⒋ } 48^{\prime} 40.1^{\prime \prime}\end{array}$ & $\begin{array}{l}\text { Citrus sp., Cocos nucifera, Dimocarpus } \\
\text { longan, Nephelium lappaceum }\end{array}$ & & & & $\sqrt{ }$ & $\sqrt{ }$ & $\sqrt{ }$ & $\sqrt{ }$ & & $\sqrt{ }$ & & \\
\hline & 23 & $\begin{array}{l}\mathrm{N} 04^{\circ} 42^{\prime} 48.2^{\prime \prime} \\
\mathrm{E} 114^{\circ} 47^{\prime} 36.0^{\prime \prime}\end{array}$ & Secondary jungle & $\sqrt{ }$ & $\sqrt{ }$ & $\sqrt{ }$ & $\sqrt{ }$ & $\sqrt{ }$ & $\sqrt{ }$ & $\sqrt{ }$ & & $\sqrt{ }$ & & \\
\hline
\end{tabular}


Reference of common names for the scientific names

Acacia sp.

Annona muricata

Archidendron pauciflorum

Artocarpus heterophyllus

Artocarpus integer

Averrhoa carambola

Canarium odontophyllum

Capsicum sp.

Carica papaya

Citrus maxima

Citrus sp.

Cocos nucifera

Cucmis sativa

Dimocarpus longan

Durio sp.

Elaies guineensis

Hylocereus sp.

Lansium domesticum

Litsea garciae

Luffa sp.

Mangifera sp.

Mangiferae pajang

Manilkara zapota

Momordica charantia

Musa sp.

Nephelium lappaceum

Pangium edule

Piper nigrum

Psidium sp.

Solanum melongena

Syzygium samarangense

Theobroma cocoa

Vigna unguiculata sesquipedalis

Vitex pinnata

Zea mays
Acacia

Soursop

Jering

Jackfruit

Cempedak

Star fruit

Dabai

Chilli

Papaya

Pamelo

Citrus

Coconut

Cucumber

Longan

Durian

Oil palm

Dragon fruit

Langsat

Engkalak

Luffa

Mango

Membangan

Chiku

Bittergourd

Banana

Rambutan

Kepayang

Pepper

Guava

Brinjal

Wax apple

Cocoa

Long bean

Kulimpapa

Maize 